\title{
H-, He-like recombination spectra - III. $n$-changing collisions in highly excited Rydberg states and their impact on the radio, IR, and optical recombination lines
}

\author{
F. Guzmán, ${ }^{1 \star}$ M. Chatzikos, ${ }^{1}$ P. A. M. van Hoof, ${ }^{2}$ Dana S. Balser, ${ }^{3}$ M. Dehghanian, ${ }^{1}$ \\ N. R. Badnell ${ }^{4}$ and G. J. Ferland ${ }^{1}$ \\ ${ }^{1}$ Department of Physics and Astronomy, University of Kentucky, Lexington, KY 40506, USA \\ ${ }^{2}$ Royal Observatory of Belgium, Ringlaan 3, B-1180 Brussels, Belgium \\ ${ }^{3}$ NRAO, Charlottesville, VA 22903-2475, USA \\ ${ }^{4}$ Department of Physics, University of Strathclyde, Glasgow G4 ONG, UK
}

Accepted 2019 March 14. Received 2019 March 13; in original form 2019 February 5

\begin{abstract}
At intermediate to high densities, electron (de-)excitation collisions are the dominant process for populating or depopulating high Rydberg states. In particular, the accurate knowledge of the energy changing ( $n$-changing) collisional rates is determinant for predicting the radio recombination spectra of gaseous nebula. The different data sets present in the literature come either from impact parameter calculations or semi-empirical fits and the rate coefficients agree within a factor of 2 . We show in this paper that these uncertainties cause errors lower than 5 percent in the emission of radio recombination lines of most ionized plasmas of typical nebulae. However, in special circumstances where the transitions between Rydberg levels are amplified by maser effects, the errors can increase up to 20 per cent. We present simulations of the optical depth and $\mathrm{H} n \alpha$ line emission of active galactic nuclei broad-line regions and the Orion Nebula Blister to showcase our findings.
\end{abstract}

Key words: atomic data-atomic processes-masers-H II regions-radio lines: generalsubmillimetre: general.

\section{INTRODUCTION}

Radio observations are an important tool in astronomy as the spectrum is not affected by dust extinction. Radio recombination lines (RRL) coming from highly excited Rydberg levels are used to determine the temperatures and densities of gaseous nebulae (see e.g. Osterbrock \& Ferland 2006), the metallicity structure of the Galaxy (Balser et al. 2011, 2015), to survey the H II regions of our Galaxy (Anderson et al. 2018) and determine their structure (Poppi et al. 2007; Anderson et al. 2015), to probe extragalactic active galactic nuclei (AGNs) and startburst galaxies (Scoville \& Murchikova 2013), to study the photodissociation regions (Luisi et al. 2017), and to study the diffuse ionized gas in H II regions and the diffuse clouds of the cold neutral medium (Morabito et al. 2014).

In particular, hydrogen and helium RRL can be used to obtain the electron temperature $T_{\mathrm{e}}$. If the levels involved in the line transition are close to local thermodynamic equilibrium (LTE),

^E-mail: francisco.guzman@uky.edu
$T_{\mathrm{e}}$ can be obtained directly from the ratio of the line brightness temperature to the continuum brightness temperature (Rohlfs \& Wilson 2000). However, non-LTE effects can be important for RRL (Brown, Lockman \& Knapp 1978). Likewise, stimulated emission (maser effects) and pressure broadening effects can alter the equilibrium of lines (Shaver 1980). In these cases, modelling of the level populations using collisional radiative (CR) modelling (Dupree \& Goldberg 1970; Brocklehurst \& Seaton 1972; Burgess \& Summers 1976) is needed to obtain the correct line intensities. $\mathrm{CR}$ modelling is subject to the availability and accuracy of the fundamental atomic data describing the processes between the different particles of the emitting gas. The highest uncertainties come from the collisional (de-)excitation, which can be divided into two types of interaction: energy changing and angular momentum changing. In the previous two papers of this series, devoted to investigate and assess these collisional data, which have a high impact on astronomical observations (Guzmán et al. 2016, 2017, hereafter Paper I and Paper II), we showed how the disparities in the theoretical values of $l$-changing collisions cross-sections can affect the emissivities of the optical recombination lines. $l$-changing collisions are mainly produced by ion impact and alter the angular 
momentum of the target electron by Stark mixing without changing its energy (Pengelly \& Seaton 1964). As shown in Paper I and Paper II, at high Rydberg states, $l$-changing collisions are so fast that they populate the $l$ subshells statistically, and differences of one order of magnitude between the values of the rate coefficients are not important. However, as noted by Salgado et al. (2017), the low densities of the cold neutral medium make the Stark effect interactions of $l$-changing collisions important at the high levels involved in RRL.

This paper is the third of the series and is focused on electron impact excitations changing the principal quantum number $n$ of the target electron. These energy changing electron collisions redistribute the electronic population of the high Rydberg levels of ions and atoms towards LTE. However, many of the Rydberg states will remain in non-LTE. These effects will mainly depend on the accuracy of the electron-impact excitation rate coefficients, which can significantly influence the derived opacities and the temperature diagnostics obtained with RRL. Unfortunately there are no precise quantum calculations for collisional excitation of high Rydberg levels. R-matrix calculations for $n$-changing collisional data for hydrogen- and helium-like (H-like and He-like) iso-sequences reach up to $n=5$ (Anderson et al. 2000; Bray et al. 2000; Ballance et al. 2006; Ralchenko et al. 2008; Griffin \& Ballance 2009). Analytic formulas using the Born approximation (Lebedev \& Beigman 1998) and fitting formulas from theoretical and experimental data (Fujimoto 1978; Vriens \& Smeets 1980) have been used for transitions with higher $n$.

In this paper, we analyse the impact of the different approaches for $n$-changing electron-impact excitation cross-sections on the Rydberg populations of $\mathrm{H}$ - and He-like ions and on the RRL. Our main goal is to identify the best data and quantify how the determination of the physical parameters derived from the observed RRL can be compromised by the uncertainty in the $n$-changing collisions. In Section 2, the different approximations are discussed and compared. In Section 3, we discuss how the different values of $n$-changing rate coefficients translate to the population densities and the predicted RRL intensities and opacities. In that section, we analyse a simple slab of irradiated gas using the spectral simulation code CLOUDY, last described by Ferland et al. (2017). The uncertainties on the excitation rates at high Rydberg levels could affect the lower levels by cascading decay, and thus compete with the errors from the collisional data for low-lying levels. We discuss these effects on the uncertainty of the predicted emissivities of optical recombination lines in Section 4. In Section 5.1 and 5.2 we apply our analysis to models of the broad-line regions (BLRs) in AGN and the Orion nebula emission, respectively. Finally, we summarize our results in the conclusions.

\section{RYDBERG COLLISIONAL EXCITATION THEORIES}

Electron-impact excitation between high $n$ Rydberg $(n \geq 5)$ levels have received considerably less attention than between low-lying $n$ shells due to a number of reasons. First, the low-lying transitions in the optical part of the spectrum are much brighter than the radio lines and less affected by pressure broadening. Line ratios are routinely used in the optical to obtain temperatures and densities. Second, dust absorption and scattering peaks at the mid-infrared and is still big at the far-infrared (Osterbrock \& Ferland 2006) making intermediatehigh Rydberg levels transitions unobservable in dusty environments. Third, the numerical and computational limitations of the theoretical methods prevent accurate quantum calculations for transitions to

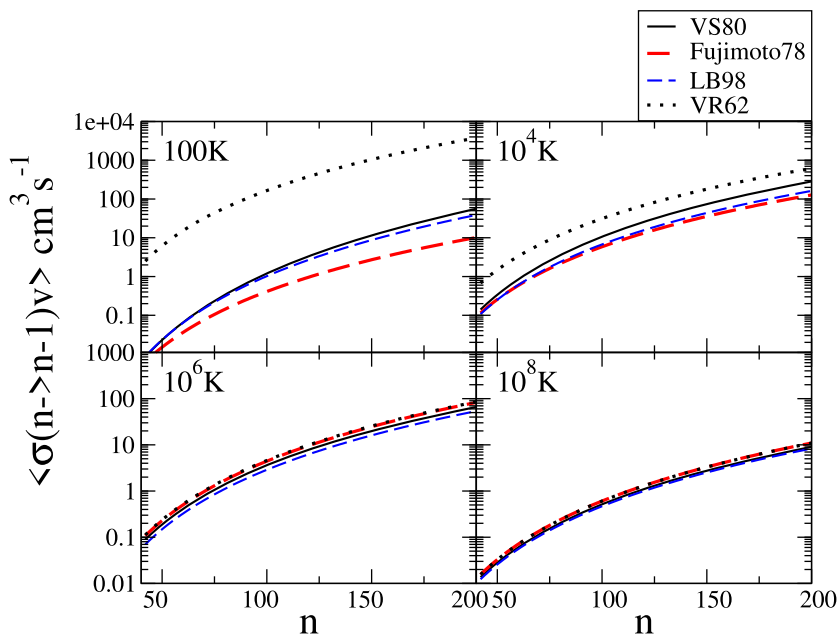

Figure 1. Effective rate coefficients for $n \rightarrow n-1$ electron-impact transitions as a function of $n$ for different electron temperatures (indicated at the top left of each panel). VS80: Vriens \& Smeets (1980); LB98: Lebedev \& Beigman (1998); Fujimoto78: Fujimoto (1978); VR62: van Regemorter (1962).

higher levels. For example, in most R-matrix calculations, including higher levels requires the appropriate inclusion of pseudo-states to correctly cover excitation in the close coupling terms. The number of pseudo-states needed will grow with the inclusion of more levels Ballance et al. (2006) show how including the coupling to pseudostates modified their results between 40 percent and 60 percent. Finally, in very low density environments, such as in the interstellar medium (ISM), low levels recombine faster and the Rydberg levels eventually decay radiatively to lower levels. At high densities, collisions are so fast that the levels are expected to be in LTE independently of the value of the collisional transition probability, so the actual theory is unimportant. However, there is a wide number of astrophysical circumstances where the Rydberg levels can be in non-LTE and their populations driven by the rates of electron impact collisions (see e.g. Sections 3 and 5).

\subsection{Collisional excitation data sets}

Maxwell-averaged collisional effective rate coefficients for the dominant $n \rightarrow n-1$ de-excitations, where $n$ is the principal quantum number of Rydberg states, are plotted in Figs 1 and 2 for neutral atoms and for $T_{\mathrm{e}}=100-10^{8} \mathrm{~K}$ as a function of the upper level main quantum number $n$ and the electron temperature, respectively. While the curves have a similar shape (with the exception of the VR62 set explained later in this section, they differ from 30 per cent at high temperatures to 60 per cent at $T_{\mathrm{e}} \sim 10^{4}-10^{5} \mathrm{~K}$. In this paper we investigate whether these uncertainties could impact predictions of astrophysical interest.

\subsubsection{VR62}

van Regemorter (1962) obtained new values for the experimentally averaged Gaunt factor $\bar{g}$ approximation proposed by Seaton (1959). The effective rates for de-excitation proposed by this model are

$q_{n^{\prime} \rightarrow n}=\frac{20.60 \lambda^{3} A_{n n^{\prime}}}{\sqrt{T_{\mathrm{e}}}} P(\epsilon) \quad \mathrm{cm}^{3} \mathrm{~s}^{-1}$, 


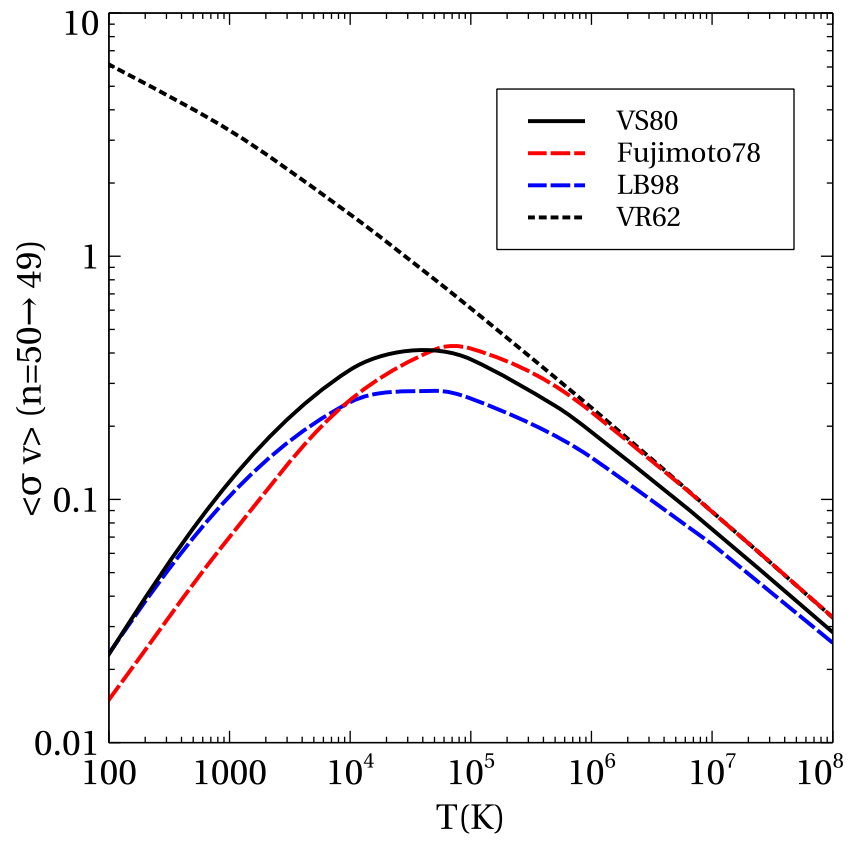

Figure 2. Effective rate coefficients for $n \rightarrow n-1$ electron impact deexcitation for $n=50-49$ as a function of the electron temperature. Legend labels as in Fig. 1.

where $T_{\mathrm{e}}$ is the electron temperature in $\mathrm{K}, \lambda=\frac{h c}{\Delta E_{n n^{\prime}}}(\mathrm{cm})$, with $h$ the Planck constant and $c$ the speed of light, $\Delta E_{n n^{\prime}}$ is the $n^{\prime}$ $\rightarrow n$ transition energy, $A_{n n^{\prime}}$ is the Einstein coefficient, $\epsilon=\frac{\Delta E_{n n^{\prime}}}{k T_{\mathrm{e}}}$, and

$P(\epsilon)=\int_{0}^{\infty} \bar{g}(x) \exp \left(-\epsilon x^{2}\right) \mathrm{d}\left(\epsilon x^{2}\right)$,

with $x=\sqrt{\frac{m_{\mathrm{e}} v^{2}}{\Delta E_{n n^{\prime}}}}$ (so $\epsilon x^{2}=\frac{m_{\mathrm{e}} v^{2}}{k T_{\mathrm{e}}}$ ), $m_{\mathrm{e}}$ the rest mass of the electron, $v$ the frequency of the transition $n \rightarrow n^{\prime}$, and $\bar{g}(x)$ a Gaunt factor (see e.g. Seaton 1959) obtained from fitting experimental values. van Regemorter (1962) provides values for the function $P(\epsilon)$ of equation (2) in his table 2. He used the Born approximation together with experimental results to obtain the functions $\bar{g}(x)$. We have interpolated these values to calculate the dotted curve in Figs 1 and 2. It is important to note that van Regemorter (1962) fitted his Gaunt factors to transitions between low-lying levels of atoms and ions (see fig. 1 of his paper) relevant for UV observations of the solar corona. The VR62 results greatly overestimate later calculations for high Rydberg levels at low and intermediate temperatures and should not be used at low temperatures. We have included them here for comparison purposes. These results are in better agreement with the semi-empirical formulas of Fujimoto78 at high energies, where the correct asymptotic behaviour of $T^{-1 / 2}$ is ensured. This is shown in Fig. 2 (more details in Section 2.1.2).

\subsubsection{Fujimoto78}

Fujimoto (1978), in an internal report, integrated experimental and theoretical cross-section values fitted by Johnson \& Hinnov (1969) to a fitting formula for rate coefficients. These authors used measurements of population densities in an experimental plasma at the C stellarator (Sinclair et al. 1965) and compared with the solution of the CR balance equations for each level to deduce the excitation cross-sections. The experiments were done for a range of electron temperatures between 0.04 and $1 \mathrm{eV}$ (464 and $11604 \mathrm{~K}$ ) and electron densities between $10^{11}$ and $3 \times 10^{13} \mathrm{~cm}^{-3}$. Asymptotic forms from the impact parameter approximation (Seaton 1962) were used for higher temperatures. The fits of Fujimoto (1978) have been recommended by Ralchenko et al. (2008) for helium transitions with upper and lower $n>5$. The de-excitation effective coefficients are

$$
\begin{aligned}
q_{n^{\prime} \rightarrow n}= & \mathrm{e}^{-\epsilon} K\left[\frac{\ln (1+\delta)-\mathrm{e}^{(1+\delta) \epsilon} E_{1}(-(1+\delta) \epsilon)}{\epsilon}\right] \\
& -\frac{\mathrm{e}^{-2 \beta\left(f_{n n^{\prime}} \mathrm{I}_{\mathrm{H}} / \Delta E_{n n^{\prime}}\right)^{-\gamma}}}{\beta\left(f_{n n^{\prime}} \mathrm{I}_{\mathrm{H}} / \Delta E_{n n^{\prime}}\right)^{-\gamma}+\epsilon} \\
& \times\left\{\ln (1+\delta)+\mathrm{e}^{(1+\delta)\left[\beta\left(f_{n n^{\prime}} \mathrm{I}_{\mathrm{H}} / \Delta E_{n n^{\prime}}\right)^{-\gamma}+\epsilon\right]}\right. \\
& \left.\times E_{1}\left((1+\delta)\left[\beta\left(f_{n n^{\prime}} \mathrm{I}_{\mathrm{H}} / \Delta E_{n n^{\prime}}\right)^{-\gamma}+\epsilon\right]\right)\right\},
\end{aligned}
$$

where

$K=8\left(\frac{\mathrm{I}_{\mathrm{H}}}{k T_{\mathrm{e}}}\right)^{3 / 2} \sqrt{\pi} \alpha c a_{0}^{2} f_{n n^{\prime}}$.

In equations ( 3 ) and (4) $\mathrm{I}_{\mathrm{H}}$ is the Rydberg energy, $f_{n n^{\prime}}$ is the oscillator strength of the $n-n^{\prime}$ transition, $\alpha$ is the fine structure constant, $a_{0}$ is the Bohr radius, $\epsilon=\frac{\Delta E_{n n^{\prime}}}{k T_{\mathrm{e}}}$ as in equations (1) and (2), $E_{1}(x)$ is the first exponential integral (see Abramowitz \& Stegun 1965) and $\beta=0.5, \gamma=0.7$, and $\delta=0.2$ are fitting constants. Since Johnson \& Hinnov (1969) use results from the impact parameter approximation (Seaton 1962), and these results have been also used by van Regemorter (1962) for his $\bar{g}$ approach, it is not surprising that Fujimoto and Van Regemorter data sets coincide in the limit of the high electron temperatures that can be seen in Fig. 1, and specially in Fig. 2. Johnson \& Hinnov (1969) report high uncertainties in their cross-sections for high $n$ because these levels are closer to LTE, and the value of the cross-sections does not have an important influence in the populations.

\subsubsection{VS80}

Vriens \& Smeets (1980) fit experimental results for intermediatehigh energies using several theoretical and experimental works available at the time. Their excitation coefficients are mainly based on the semi-empirical formulas of Gee et al. (1976). These authors recommend their results for $10^{6} \mathrm{~K} / n^{2}<T_{\mathrm{e}} \ll 3 \times 10^{9} \mathrm{~K}$. Vriens \& Smeets (1980) overcame this limitation by using their own extrapolation at lower energies, simultaneously fitting experimental and theoretical results, including Percival \& Richards (1976) semiempirical formulae, Johnson (1972) Bethe-Born approximation, and available experimental data (see references within Vriens \& Smeets 1980). The final de-excitation rate coefficients are given by

$$
\begin{aligned}
q_{n^{\prime} \rightarrow n}= & 4.33 \times 10^{-8} \frac{g_{n}}{g_{n^{\prime}} \frac{\left(\frac{k T_{\mathrm{e}}}{\mathrm{I}_{\mathrm{H}}}\right)^{1 / 2}}{\mathrm{I}_{\mathrm{H}}}+\Gamma_{n n^{\prime}}} \\
& \times\left[\mathcal{A}_{n n^{\prime}} \ln \left(\frac{0.3 k T_{\mathrm{e}}}{\mathrm{I}_{\mathrm{H}}}+\Delta_{n n^{\prime}}\right)+\mathcal{B}_{n^{\prime} n}\right] \mathrm{cm}^{3} \mathrm{~s}^{-1},
\end{aligned}
$$


where $g_{n}$ and $g_{n^{\prime}}$ are the statistical weights of the states $n$ and $n^{\prime}$, and

$$
\begin{aligned}
\Gamma_{n n^{\prime}}= & \ln \left(1+\frac{n^{3} k T_{\mathrm{e}}}{\mathrm{I}_{\mathrm{H}}}\right)\left[3+11\left(\frac{\left|n^{\prime}-n\right|}{n}\right)^{2}\right] \\
& \times\left(6+1.6 n^{\prime}\left|n^{\prime}-n\right|+0.3\left|n^{\prime}-n\right|^{-2}\right. \\
& \left.+0.8 n^{3 / 2}\left|n^{\prime}-n\right|^{-1 / 2}|| n^{\prime}-n|-0.6|\right)^{-1} \\
\Delta_{n n^{\prime}}= & \exp \left(\frac{\mathcal{B}_{n n^{\prime}}}{\mathcal{A}_{n n^{\prime}}}\right)+\frac{0.06\left|n^{\prime}-n\right|^{2}}{n^{\prime} n^{2}} \\
\mathcal{A}_{n n^{\prime}}= & \left(\frac{2 \mathrm{I}_{\mathrm{H}}}{\Delta E_{n n^{\prime}}}\right) f_{n n^{\prime}} \\
\mathcal{B}_{n n^{\prime}}= & \frac{4 \mathrm{I}_{\mathrm{H}}^{2}}{n^{\prime 3}}\left(\frac{1}{\Delta E_{n n^{\prime}}^{2}}+\frac{4 E_{n}}{3 \Delta E_{n n^{\prime}}^{3}}+c_{n} \frac{E_{n}^{2}}{\Delta E_{n n^{\prime}}^{4}}\right) \\
c_{n}= & \frac{1.4 \ln (n)}{n}-\frac{0.7}{n}-\frac{0.51}{n^{2}}+\frac{1.16}{n^{3}}-\frac{0.55}{n^{4}},
\end{aligned}
$$

and $E_{n}$ is the ionization potential of the shell $n$. The remaining symbols have the same meaning as in equations (3) and (4).

We have checked that the rate coefficients from Gee et al. (1976) agree with Vriens \& Smeets (1980) within 15 percent over their range of validity. Additionally, Percival \& Richards (1978) modified Gee et al. (1976) coefficients to include charge dependence.

\subsubsection{LB98}

Lebedev \& Beigman (1998) use the semiclassical straight-trajectory Born approximation to provide $a b$ initio excitation rate coefficients for Rydberg atoms. The de-excitation formula for $n^{\prime} \rightarrow n$ is

$$
\begin{aligned}
q_{n^{\prime} \rightarrow n} & =\frac{g_{n}}{g_{n^{\prime}}} \exp \left(\frac{\Delta E_{n n^{\prime}}}{k T_{\mathrm{e}}}\right) q_{n \rightarrow n^{\prime}} \\
& =\frac{g_{n}}{g_{n^{\prime}}} 2 \sqrt{\pi} a_{0}^{2} \alpha c n\left[\frac{n^{\prime}}{Z\left(n^{\prime}-n\right)}\right]^{3} \frac{f(\theta) \varphi}{\sqrt{\theta}},
\end{aligned}
$$

where

$$
\begin{aligned}
\varphi= & \frac{2 n^{\prime 2} n^{2}}{\left(n^{\prime}+n\right)^{4}\left(n^{\prime}-n\right)^{2}}\left[4\left(n^{\prime}-n\right)-1\right] \exp \left(\frac{E_{n}}{k T_{\mathrm{e}}}\right) E_{1}\left(\frac{E_{n}}{k T_{\mathrm{e}}}\right) \\
& +\frac{8 n^{3}}{\left(n^{\prime}+n\right)^{2}\left(n^{\prime}-n\right) n^{2} n^{\prime 2}}\left(n^{\prime}-n-0.6\right)\left(\frac{4}{3}+n^{2}\left(n^{\prime}-n\right)\right) \\
& \times\left[1-\frac{E_{n}}{k T_{\mathrm{e}}} \exp \left(\frac{E_{n}}{k T_{\mathrm{e}}}\right) E_{1}\left(\frac{E_{n}}{k T_{\mathrm{e}}}\right)\right] \\
f(\theta)= & \frac{\ln \left(1+\frac{n \theta}{Z\left(n^{\prime}-n\right) \sqrt{\theta}+2.5}\right)}{\ln \left(1+\frac{n \sqrt{\theta}}{Z\left(n^{\prime}-n\right)}\right)}
\end{aligned}
$$

$\theta=\frac{k T_{\mathrm{e}}}{Z^{2} \mathrm{I}_{\mathrm{H}}}$,

and $\alpha$ and $Z$ are the fine structure constant and the core charge of the target ion or atom. While VS80, Fujimoto78, and VR62 formulas are theoretical formulas fitted to experimental results, LB98 is a semiclassical treatment (impact parameter) of the Born approximation and involves no fit to experimental results. Lebedev \& Beigman (1998) note that the straight trajectories approximation breaks at low energies and when the target ions have high charges. Coulomb trajectories should be assumed in these cases. Because of that, we expect an underestimation of the rates obtained with this formula at low collision energies and $Z>1$. Fujimoto78 and VS80 formulas have the same shape at low temperatures, but they are intended only for atoms. A study of the collisional rates for higher charges is beyond the scope of this paper and will be the object of future work.

\subsection{Comparison of the collisional excitation rate coefficients}

The curves in Fig. 1 are mostly parallel for all the range of $n \rightarrow n-$ 1 collisional transitions considered here. In Fig. 2 LB98, VS80, and Fujimoto78 data sets have a similar dependence with the temperature. The $\bar{g}$ approximation used by VR62 was originally conceived for high energies and has the correct $\mathrm{T}^{-1 / 2}$ dependence at high temperatures, failing to give the correct low energy behaviour. This is a known fact of the $\bar{g}$ approach. Fujimoto 78 formula has the same high energy dependence as VR62. However, there is reasonable doubt over the experimental rate coefficients at temperatures above the range probed by the experiments of Johnson \& Hinnov (1969). These authors use Seaton (1962) approach. Furthermore, levels at very high $n$ might be in LTE for the high electron densities of the experiments producing inaccurate values of the rate coefficients. A visual inspection of fig. 5 of Vriens \& Smeets (1980) shows that the Gee et al. (1976) values and the VS80 extrapolations do not differ by more than a factor $\sim 2$. This is similar to the difference between VS80 and LB98 and Fujimoto78. Salgado et al. (2017) compare the values of Vriens \& Smeets (1980) with the rate coefficients given by Gee et al. (1976) for low temperatures obtaining differences of less than 20 percent. LB98 ab initio rate coefficients are supported by the experimental fits included in the VS80 and Fujimoto78 data sets (Fig. 2) even at low temperatures, indicating that the straight-trajectory approximation is good in this range at least for neutral targets. The LB98 calculations do not rely on extrapolations from previous experimental fittings and might be more accurate.

\subsection{1 $\Delta \mathrm{n}>1$}

Although (de-)excitation collisions with $\Delta n=1$ are dominant, collisions with higher $\Delta n$ can contribute to (de)populate high Rydberg levels. While Fujimoto78 and VR62 depend on $\Delta n$ through $\Delta E_{n n^{\prime}}$, LB98 and VS80 have a more complicated dependence. Rate coefficients for $\Delta n<4$ are compared on the four panels of Fig. 3 for collisional de-excitation transitions depopulating $n=50$ (lefthand panels) and populating $n=49$ (right-hand panels). $\Delta n>$ 1 collisions contribute to the populating and depopulating rates appreciably. For example, at $T_{\mathrm{e}}=10^{4} \mathrm{~K}, \Delta n=2$ depopulating rate coefficients for $n=50$ are $\sim 14$ per cent of $\Delta n=1$ collisional transitions for VS80 data and $\sim 9$ per cent for LB98, while $\Delta n=3$ contributes another $\sim 4$ per cent for VS80 and $\sim 2$ per cent to LB98. The final contribution of $1<\Delta n<4$ is then $\sim 18$ percent for VS80 and $\sim 11$ per cent for LB98 increasing the difference between the final (de)populating rates obtained using each data set. $\Delta n>$ 1 collisions are included in the CLOUDY simulations shown in this paper unless specified otherwise. 


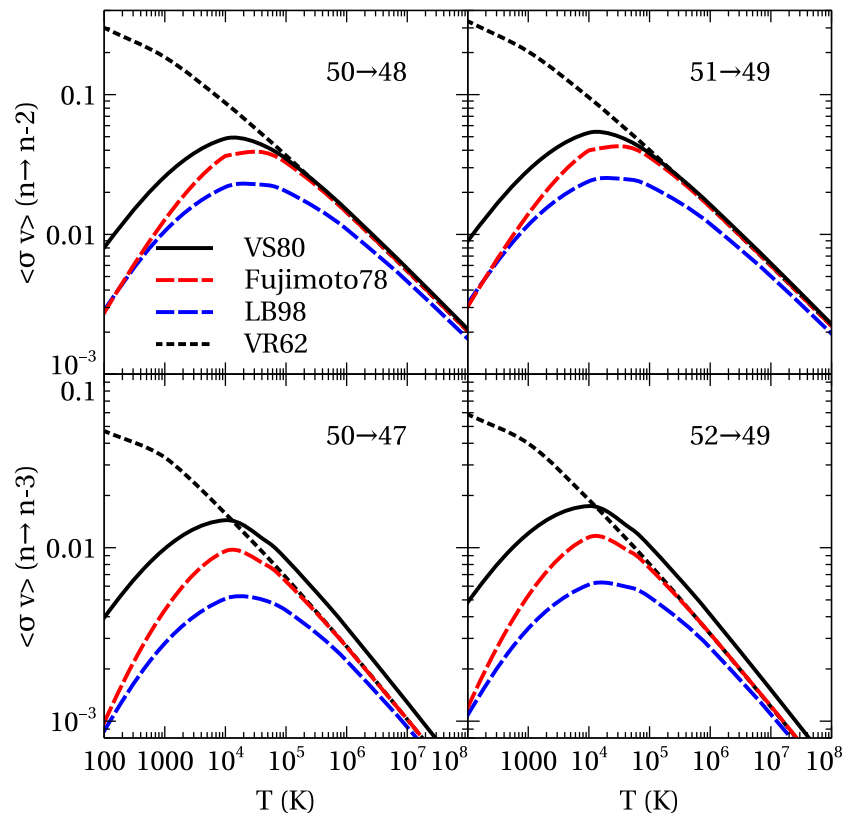

Figure 3. Effective rate coefficients for $n \rightarrow n-2$ and $n \rightarrow n-3$ electron impact transitions as a function of temperature for different $n$ indicated at the top right corner of each panel. Legend labels as in Fig. 1.

\section{EMISSION OF RADIO RECOMBINATION LINES}

RRL of H-like and He-like elements are produced by transitions between high $n$-shells Rydberg levels. At high enough $n, l$-changing collisional transitions overcome radiative decay and bring the $l$ subshells to equilibrium. At this point we can consider the populations of the $l$-shells to be statistically distributed as

$n_{n l}=\frac{2 l+1}{n^{2}} n_{n}$

The total radiative lifetime is $\tau_{n l}=A_{n l}^{-1} \propto n^{5}$, where $A_{n l}$ is the total spontaneous decay rate out of level $n l$. The collisional redistribution time can be calculated as $\tau_{l l^{\prime}}^{\text {coll }}=\left(n_{i} q_{l l^{\prime}}\right)^{-1}$, where $n_{i}$ is the density of heavy ions ${ }^{1}$ and $q_{l l^{\prime}}$ is the collisional effective coefficient for the $n l \rightarrow n l^{\prime}$ transition. If $l \ll n, q_{l l^{\prime}} \sim n^{4}$ (Pengelly \& Seaton 1964). The critical density where $l$-changing collisions rates are equal to radiative transitions is defined as $n^{\text {crit }}=1 /\left(\tau_{n l} q_{l l^{\prime}}\right)$ and will depend on $n$ as $\sim n^{-9}$. For $n \sim 30$ in hydrogen at $T=10^{4} \mathrm{~K}, n^{\text {crit }} \simeq 30 \mathrm{~cm}^{-3}$ and for $n \sim 50$ and $n^{\text {crit }} \simeq 0.6 \mathrm{~cm}^{-3}$. Typical densities of $\mathrm{H}$ II regions are of $n_{\mathrm{H}} \sim 10^{3}-10^{4} \mathrm{~cm}^{-3}$, while diffuse atomic gas have $n_{\mathrm{H}} \sim$ $50 \mathrm{~cm}^{-3}$. We can assume that levels with $n>50$ are statistically populated in the $l$-subshells as in equation (9). For the much lower ISM, intergalactic or intracluster densities, this assumption is not valid and $l$-subshell populations need to be considered. Thus, the equilibrium of the high Rydbergs is determined by the $n$-changing collisions, where transitions $n \rightarrow n-1$ are dominant. At high enough $n$, collisions are frequent and populations will be driven to LTE.

\footnotetext{
${ }^{1}$ Heavy ions are more effective than electrons in intrashell collisions
} changing angular momentum (Pengelly \& Seaton 1964).

\subsection{Rydberg emissivities}

The local emissivities for $n \rightarrow n^{\prime}$ transitions are given by

$j_{n n^{\prime}}=n_{n} \frac{A_{n n^{\prime}}}{4 \pi} h \nu$,

where $A_{n n^{\prime}}$ are the $l$-averaged Einstein coefficients for $n \rightarrow n^{\prime}$ transitions, and from (9):

$A_{n n^{\prime}}=\sum_{l^{\prime}=0}^{l^{\prime}=n^{\prime}-1} \sum_{l=0}^{l=n-1} \frac{(2 l+1)}{n^{2}} A_{n l, n^{\prime} l^{\prime}}$.

The population can be obtained by solving the collisional-radiative system (see e.g. Brocklehurst 1970a; Osterbrock \& Ferland 2006):

$$
\begin{aligned}
n_{+} n_{e} \alpha_{n}+\sum_{n^{\prime}>n} n_{n^{\prime}} A_{n^{\prime} n}+\sum_{n^{\prime}} n_{n^{\prime}} j_{v} B_{n^{\prime} n}+\sum_{i} \sum_{n^{\prime}} n_{i}^{\mathrm{col}} n_{n^{\prime}} q_{n^{\prime} n} \\
=n_{n}\left[\sum_{n^{\prime}<n} A_{n n^{\prime}}+\sum_{n^{\prime}} j_{v} B_{n n^{\prime}}\right. \\
\left.+\Gamma_{n}+\sum_{i} \sum_{n^{\prime}} n_{i}^{\mathrm{col}} q_{n n^{\prime}}+\sum_{i} n_{i}^{\mathrm{col}} S_{n}^{\mathrm{ion}}\right] .
\end{aligned}
$$

Here, $\alpha_{n}=\alpha_{n}^{\mathrm{rad}}+\alpha_{n}^{\mathrm{DR}}+n_{e} \alpha_{n}^{3 \mathrm{~b}}$ is the total recombination to the shell $n$, composed of radiative recombination (including spontaneous and induced recombination), dielectronic recombination (in case of He-like ions), and three body recombination, respectively. $A_{n n^{\prime}}$ and $B_{n n^{\prime}}$ are the Einstein spontaneous and stimulated emission coefficients, $\Gamma_{n}$ is the photoionization integral from level $n, q_{n n^{\prime}}$ is the collisional rate coefficient $\left(\mathrm{cm}^{3} \mathrm{~s}^{-1}\right)$ of the transition from level $n$ to level $n^{\prime}, n_{+}$is the population of the parent ion before recombination, $n^{\text {col }}$ represent the density of colliders responsible for this transition (electrons or heavy particles) and $S_{n}^{\text {ion }}$ is the ionization rate coefficient from level $n$. In equation (12), radiative excitation terms are negligible for most astrophysical systems. Radiative recombination and photoionization contribute mostly to the lower lying levels, while three body recombination and collisional ionization terms are more important for the high Rydberg levels, approaching them to LTE.

It is common to express the population of the $n$-shell as a function of the departure coefficients $b_{n}$ :

$n_{n}=n_{e} n_{+}\left(\frac{h^{2}}{2 \pi m_{\mathrm{e}} k T_{\mathrm{e}}}\right)^{3 / 2} \frac{g_{n}}{2} \mathrm{e}^{\frac{E_{n}}{k T_{e}}} b_{n}=n_{n}^{\mathrm{LTE}} b_{n}$,

where $g_{n}$ is the statistical weight of the shell $n$. In LTE, $b_{n}=1$ and the populations $n_{n}$ will obey the Saha-Boltzmann law. To obtain the departure coefficients, the CR equations (12) for the populations of the ions of the gas need to be solved. Substituting equation (13), it is possible to write the equations (12) in terms of the departure coefficients:

$$
\begin{aligned}
& \left(\frac{h^{2}}{2 \pi m_{\mathrm{e}} k T_{\mathrm{e}}}\right)^{-3 / 2} \frac{\alpha_{n}}{n^{2}} \mathrm{e}^{-E_{n} / k T_{\mathrm{e}}}+\sum_{n^{\prime}>n} \frac{n^{\prime 2}}{n^{2}} \mathrm{e}^{\Delta E / k T_{\mathrm{e}}} A_{n^{\prime} n} b_{n^{\prime}} \\
& +\sum_{n^{\prime}} \frac{n^{\prime 2}}{n^{2}} \mathrm{e}^{\Delta E / k T_{\mathrm{e}}} j_{v} B_{n^{\prime} n} b_{n^{\prime}}+\sum_{n_{c}} \sum_{n^{\prime}} q_{n n^{\prime}} b_{n^{\prime}} \\
& =b_{n}\left[\sum_{n^{\prime}<n} A_{n n^{\prime}}+\sum_{n^{\prime}} j_{v} B_{n n^{\prime}}+\Gamma_{n}+\sum_{i} \sum_{n^{\prime}} n_{i}^{\mathrm{col}} q_{n n^{\prime}}\right. \\
& \left.+\sum_{i} n_{i}^{\mathrm{col}} S_{n}^{\mathrm{ion}}\right]
\end{aligned}
$$




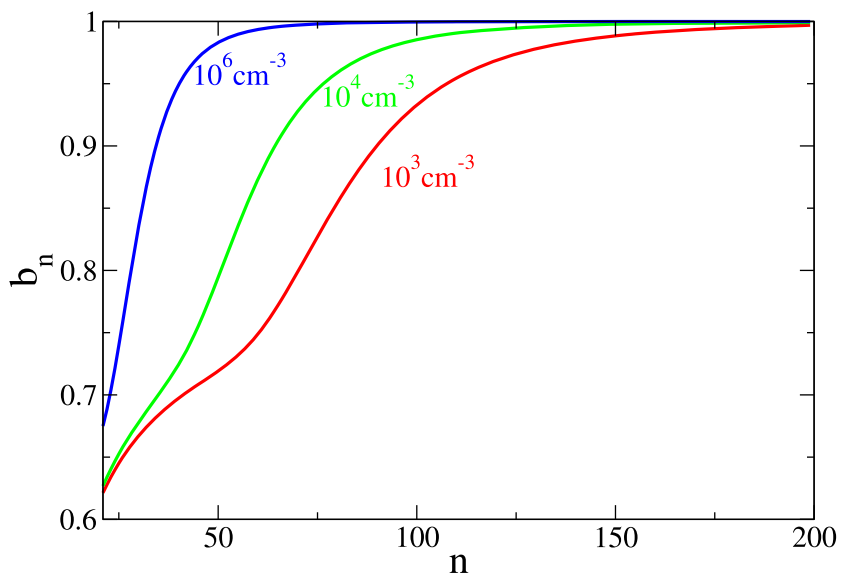

Figure 4. Departure coefficients obtained for a layer of gas illuminated by monochromatic radiation (see text for details and explanation).

where $\Delta E=E_{n^{\prime}}-E_{n}$ and we have used detailed balance in the collisional coefficients: $\mathrm{e}^{E_{n^{\prime}} / k T} g_{n^{\prime}} q_{n^{\prime} n}=\mathrm{e}^{E_{n} / k T} g_{n} q_{n n^{\prime}}$.

It is clear from equations (13) and (10) that

$j_{n n^{\prime}}=b_{n} j_{n n^{\prime}}^{\mathrm{LTE}}$,

where $j_{n n^{\prime}}^{\mathrm{LTE}}$ is the emissivity when the level of the population of the upper $n$-shell is in LTE.

Departure coefficients and populations will depend on both the radiative and collisional terms in the systems of equation (12) or (14), which are functions of the collider temperature and density. In Fig. 4, the $n$-averaged departure coefficients are plotted versus $n$ $(20<n<200)$ for different densities for the plane-parallel layer of the gas model in Section 3.2. As electron-impact collisional transition rates values increase with the principal quantum number as $n^{4}$, a lower rate than radiative lifetimes $\left(n^{5}\right)$, collisional transitions together with the three body recombination will drive the high- $n$ levels to LTE. At higher densities, progressively lower $n$-shells are in LTE. At lower densities, the contribution of collisional processes is lower, letting the population radiatively decay to lower levels.

\subsection{One layer model}

To study the different $n$-changing collisional rate formulas described in Section 2, we have simplified the problem to one illuminated layer of gas, composed either of hydrogen or of hydrogen and helium, where the abundance of the latter is $\frac{n_{\mathrm{He}}}{n_{\mathrm{H}}}=0.1$ to resemble cosmological abundances. We have illuminated this gas with monochromatic radiation of $E=2 \mathrm{Ryd}$, and forced the gas to a constant electron temperature and density. We have used a development version of the spectral code CLOUDY (Ferland et al. 2017) (nchanging branch, revision r12537), where we have implemented the different data sets, to solve the CR equations (12) and to predict the spectrum. We have used $20 \mathrm{nl}$-resolved states and unresolved, 'collapsed', levels up to $n=200$ using equation (9). We have assumed case B (Baker \& Menzel 1938) where the Lyman lines are reabsorbed by the gas which is optically thick for this wavelength. We have shut down photoionization from the $2 \mathrm{~s}$ metastable level and removed the continuous pumping. We have turned off the escape of photons following the scattering by a free electron. These operations are done to prevent our results from being affected by these effects and leading to possible misinterpretations of our analysis. Photoionization and pumping cause a redistribution of the populations of upper levels varying the intensity of the lines.
Escaping photons can reduce the ionization degree. The departure coefficients in Fig. 4 are obtained using this model for a layer of gas composed only of hydrogen. In this case we have used LB98 data set for collisional excitation of high Rydberg levels.

\subsection{Optical depths}

The emitted intensity is obtained solving the equation of radiative transfer

$\frac{\mathrm{d} I_{v}}{\mathrm{~d} s}=-\kappa_{v} I_{v}+j_{v}$,

where $I_{v}$ is the input intensity, $s$ is the path-length through the gas, and $\kappa_{v}$ is the absorption coefficient. The solution, in absence of input radiation (see e.g. Rohlfs \& Wilson 2000), is

$I_{v}=B_{v}\left(1-\mathrm{e}^{-\tau_{v}(s)}\right)$,

with

$\tau_{v}(s)=\int_{s}^{s_{0}} \kappa_{\nu} \mathrm{d} s$

the optical depth, and $B_{v}=j_{v} / \kappa_{v}$. The absorption coefficient of a recombination line $n \rightarrow n^{\prime}$ depends on the departure coefficients of the transition levels and the correction for stimulated emission (Goldberg 1966):

$\left(1-\frac{b_{n}}{b_{n^{\prime}}} \mathrm{e}^{-h v / k T}\right)$.

$\kappa_{v}$ can be expressed as

$\kappa_{v}=\beta_{n^{\prime}} b_{n^{\prime}} \kappa_{v}^{\mathrm{LTE}}$,

where $\kappa_{v}^{\mathrm{LTE}}$ and $\beta_{n}$ can be written as (Brocklehurst $\&$ Seaton 1972)

$\kappa_{v}^{\mathrm{LTE}}=n_{+} n_{e} \frac{h c^{2}}{16 \pi v}\left(\frac{h^{2}}{2 \pi m k T}\right)^{3 / 2} \frac{g_{n^{\prime}}}{k T} \mathrm{e}^{E_{j} / k T} A_{n n^{\prime}}$

$\beta_{n} \approx 1-\left(\frac{k T}{\mathrm{I}_{\mathrm{H}}}\right)\left(\frac{n^{3}}{2}\right) \frac{\mathrm{d} \ln b_{n}}{\mathrm{~d} n}$

with $v$ the frequency of the transition $n \rightarrow n^{\prime} . \beta_{n}$ is the correction of the absorption coefficient for stimulated emission and depends on both the departure coefficients and their slope with the principal quantum number. The departure coefficients, obtained by solving the CR equations (14) for a pure hydrogen gas using the different collisional data sets of Section 2, are given in Fig. 5 as a function of the principal quantum number. All curves are similar and slightly reflect the differences shown in Fig. 1.

In Fig. 5, larger excitation rate coefficients produce a marginally steeper slope in the departure coefficients due to the faster decay probabilities of the lower levels. VS80 and VR62 give similar departure coefficients despite their different rates for $\Delta n=1$ and $\Delta n=2$. The cause of that is related with the fact that the rate coefficients for $\Delta n>3$ for VR62 are lower than the VS80 ones, compensating for the differences of lower $\Delta n$ transitions. We have checked this by performing a hybrid calculation where the rate coefficients for $\Delta n>1$ are taken from VS80 fits while rates coefficients with $\Delta n=1$ are from VR62 formula. The results are plotted in the double-dot-dashed green line of Fig. 5, which is steeper than the pure VR62 and VS80. The $\beta_{n}$ factors depend on the slope of the departure coefficients through equation (20b). The $\beta_{n}$ for this single-layer model are given in Fig. 6. Population inversion, which is a condition for stimulated radiation, happens for $\beta_{n}<$ $0\left(\log \left(1-\beta_{n}\right)>0\right)$. The maximum of $\log \left(1-\beta_{n}\right)$ corresponds 


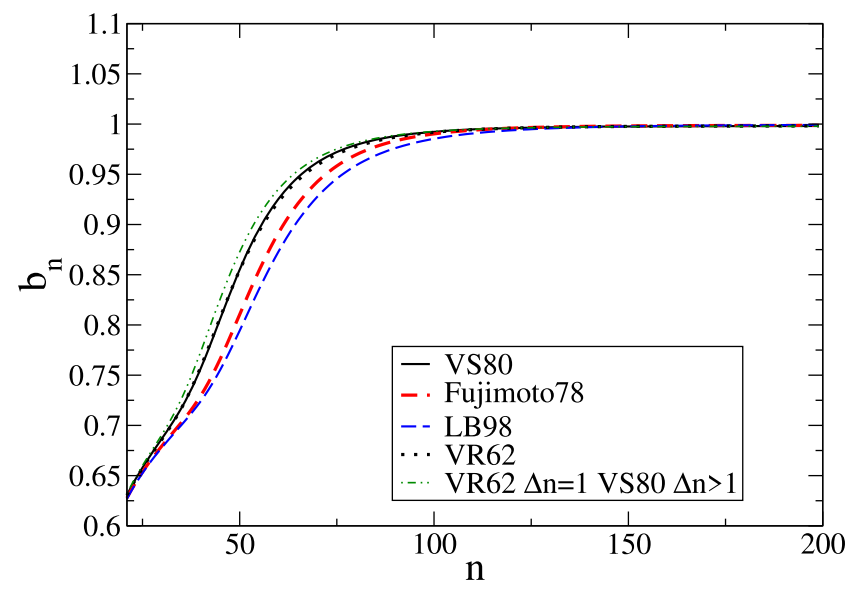

Figure 5. Departure coefficients of $\mathrm{H}^{0}$ obtained for a layer of gas $\left(T_{\mathrm{e}}=\right.$ $10^{4} \mathrm{~K}$ and $n_{e}=10^{4} \mathrm{~cm}^{-3}$ ) illuminated by monochromatic radiation. The different curves correspond to the $n \rightarrow n^{\prime}$ electron collisional data sets of Fig. 1, except for the double-dot-dashed green line where the collisional sets are taken from VR62 for $\Delta n=1$ and VS80 for $\Delta n>1$ (see text for details).

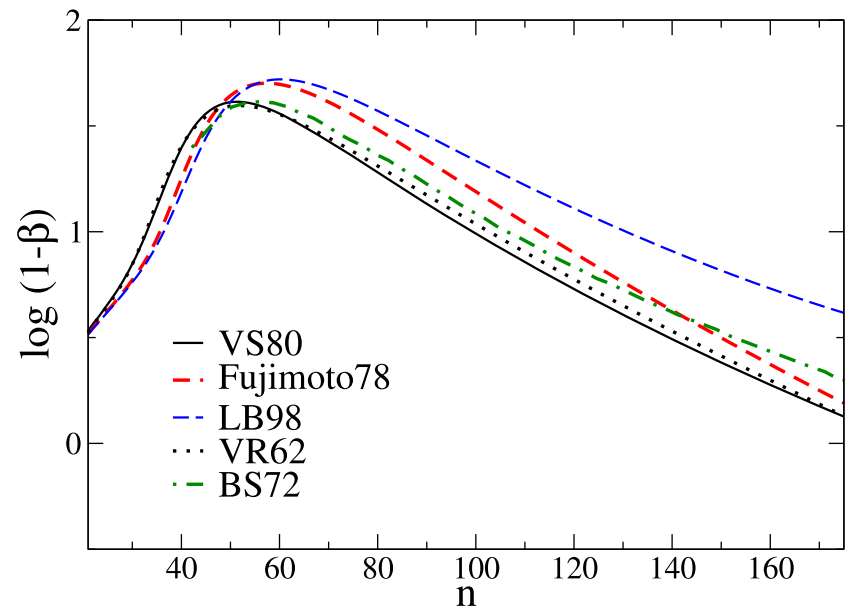

Figure 6. $\log (1-\beta)$ coefficients obtained for a layer of gas $\left(T_{\mathrm{e}}=10^{4} \mathrm{~K}\right.$ and $n_{e}=10^{4} \mathrm{~cm}^{-3}$ illuminated by monochromatic radiation). BS72 are the $\beta_{n}$ factors published in fig. 1 of Brocklehurst \& Seaton (1972). The other curves correspond to the $n \rightarrow n^{\prime}$ electron collisional data of Fig. 1 .

to the point where the population inversion is highest. This point shifts for the different data sets in correlation with the value of the collisional rate coefficients, because of the collisional distribution of the populations. As manifested in Fig. 6, $\beta_{n}$ factors turn out to be very sensitive to the differences in collisional data, which will affect the absorption coefficients, and so the optical depth and the observed spectrum. LTE is reached at $\beta_{n}=1, b_{n}=1\left(\log \left(1-\beta_{n}\right)=-\infty\right)$. All data sets tend to LTE when $n$ is high. However, big differences in the value of $\beta_{n}$ arise from the different slopes of the departure coefficients of Fig. 5, due to the different collisional (de)population probabilities. LTE will finally be ensured by the cooperation of collisional (de-)excitation and collisional ionization/three body recombination processes. It is important that our model atom is big enough to include the collisional effects of the continuum coupled to the highest levels. Their population will be transferred to lower $n$ by collisional de-excitation, which is dominant. A brief discussion

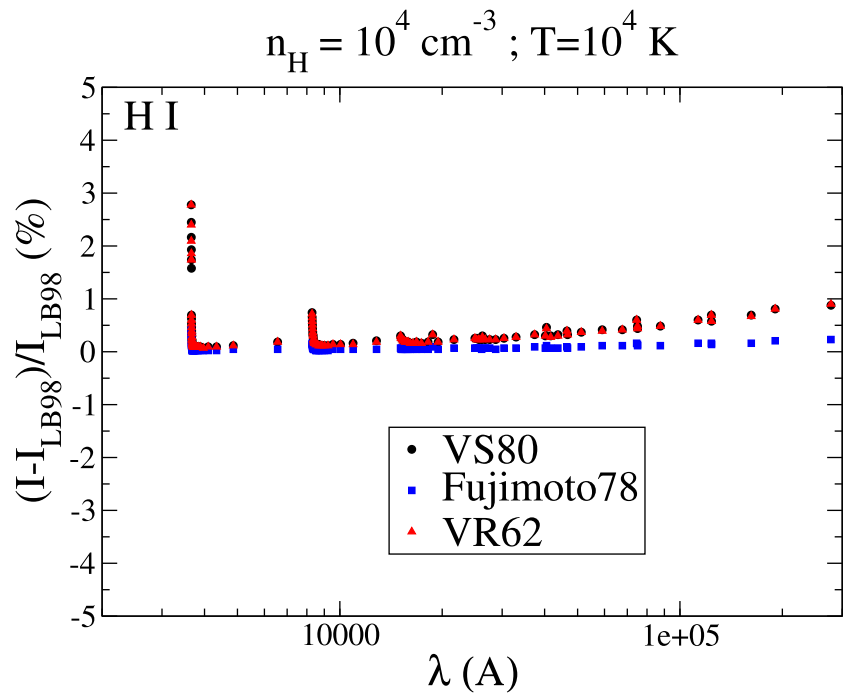

Figure 7. Ratios of $\mathrm{HI}$ emitted intensities for different data sets for a pure hydrogen gas $\left(n_{\mathrm{H}}=10^{4} \mathrm{~cm}^{-3}, T_{\mathrm{e}}=10^{4} \mathrm{~K}\right)$ illuminated by an ionizing monochromatic radiation of $2 \mathrm{Ryd}$. The intensities are normalized to the LB98 results and given in per cent units.

about the importance of the completeness of the model atom is included in appendix A.

Brocklehurst \& Seaton (1972) studied the functional dependence of the $\beta_{n}$ factors with temperature using the departure coefficients from Brocklehurst (1970a). Their model is a theoretical calculation of an homogeneous pure hydrogen nebula that is very similar to our model. Brocklehurst (1970a) used the impact parameter theory of Seaton (1962) for the dominant $\Delta n=1$ collisional transitions, and his values are close to Fujimoto78, who fitted these values (see Section 2.1.2). Their $\beta_{n}$ are the dot-dashed curve represented in Fig. 6. We could reproduce their results using the excitation rate coefficients formula from Percival \& Richards (1978). This formula give similar rate coefficients to the Brocklehurst (1970b) data sets used originally by Brocklehurst \& Seaton (1972). ${ }^{2}$

\section{IMPACT ON OPTICAL RECOMBINATION LINES}

High- $n$ excitation processes can affect optical recombination lines due to the cascade of the electrons to lower levels. In Fig. 7 we show the percentage difference of intensities of $\mathrm{HI}$ recombination lines, with principal quantum number $n=1-20$, for the data sets discussed in Section 2 with respect to LB98. We have used the one layer model discussed in Section 3.2, with pure hydrogen gas $\left(n_{\mathrm{H}}=10^{4} \mathrm{~cm}^{-3}\right.$ $\left.\simeq n_{e}\right)$, and electron temperature $\left(T_{e}=10^{4} \mathrm{~K}\right)$. Fig. 8 shows the He I recombination lines for a gas composed of hydrogen and helium as in Section 3.2. At these densities the effect of the cascading electrons is highest, as excitation and de-excitation collisions are competing with radiative processes (see Paper I). At lower densities radiative processes are dominant and at higher densities $\left(n_{\mathrm{H}} \sim 10^{10} \mathrm{~cm}^{-3}\right)$ collisional processes will bring the higher Rydberg levels to LTE, and no differences will be evident on the optical recombination lines. Excitation rate coefficients up to $n=5$ have been taken from

${ }^{2}$ Later Brocklehurst \& Salem (1977) used Gee et al. (1976) formulas in their code for the calculation of departure coefficients. 


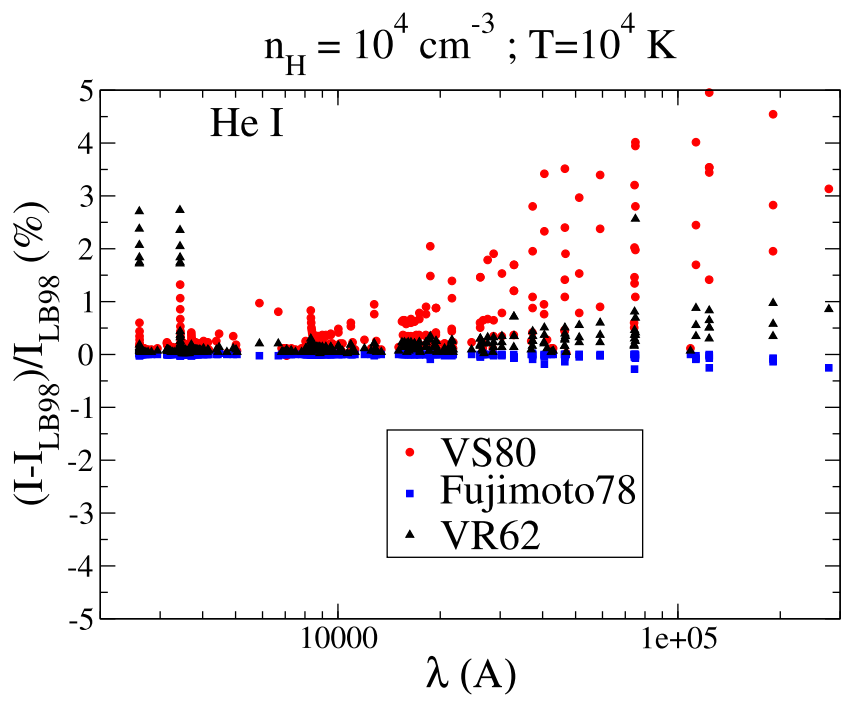

Figure 8. Ratios of He I emitted intensities for a hydrogen and helium gas (abundance $\frac{n_{\mathrm{He}}}{n_{\mathrm{H}}}=0.1, n_{\mathrm{H}}=10^{4} \mathrm{~cm}^{-3}$ and $T_{\mathrm{e}}=10^{4} \mathrm{~K}$ ) illuminated by an ionizing monochromatic radiation of $2 \mathrm{Ryd}$, enough to ionize both $\mathrm{H}$ and $\mathrm{He}$. The intensities are normalized to the LB98 results and given in per cent units.

the R-matrix calculations of Anderson et al. (2000) for hydrogen and from Bray et al. (2000) for helium in all the simulations.

In Fig. 7 the different series of lines $\Delta n=n_{u}-m$ (for a fixed principal quantum number of the lower level, $m>1$ ) can be seen. Inside each of the series, the lower wavelengths correspond to higher energy leaps where $n_{u}$ is higher, and higher wavelengths correspond to lower $\Delta n$. The deviation is bigger at the short-wavelength end of the series, because the lines come from levels that have higher $\Delta n$. Deviations also exist at the long-wavelength end of each series, due to the electron cascades from higher levels. This is seen in the Balmer series ( $m=2$, the leftmost series of values) in Fig. 7, where the greatest disagreement is at lower wavelengths, i.e. higher $\Delta n$. Deviations for $\Delta n \rightarrow 1$, are more significant for higher series $(m>$ 2 ), and grow with $m$. Note that even when the higher disagreements are produced for $n>5$ (the limit of R-matrix calculations), the lines from transitions with upper levels lower than $n_{u}=5$ are also slightly affected. In Fig. 7, the $\mathrm{H} \alpha$ and $\mathrm{H} \beta$ lines are barely affected and their intensities are $\sim 0.2$ per cent higher than the LB98 results. In Fig. 8, the situation is less clear as the lines from the singlet and triplet spin systems mix, but the different series can be readily distinguished, and the trend is similar to the H I case with $\mathrm{He}$ I $(n=$ $4 \rightarrow 2$ ) disagreeing by no more than 1 per cent.

From Figs 7 and 8, the effect of higher excitation rates in the higher Rydberg levels is to redistribute the populations, thus increasing the impact relative to LB98 data sets in the intensity of radiative cascades. Some astronomical determinations require high precision. In the case of the determination of the primordial helium abundance obtained from $\mathrm{He} \mathrm{I}$ and $\mathrm{H} \mathrm{I}$ recombination line ratios (see for example Izotov, Thuan \& Lipovetsky 1997), a precision better than 1 per cent is needed (Olive, Steigman \& Walker 2000; Izotov, Thuan \& Guseva 2014), the cascade effect could be influencing only if the lines corresponding to higher $n$ transitions of a series are used, or for wavelengths longward of $\sim 30000 \AA$ in the He I recombination spectrum.

We expect from these results that, in general, optical recombination observations will not be affected by the reported uncertainties on high Rydberg collisions. The non-ideality of the conditions of astrophysical plasmas (local velocity and density fluctuations) will smear even more the effect of the cascade from high Rydbergs making these differences practically unobservable.

\section{EFFECT ON OBSERVATIONS OF ASTRONOMICAL NEBULAE}

In order to test how the collisional uncertainties propagate to predictions, we have modelled the spectrum of two different astronomical systems. In the first case, in Section 5.1, we simulate a typical BLR illuminated by an AGN with a power-law spectral energy distribution (SED). In the second case, described in Section 5.2, we explore the effects these different collisional data have on an $\mathrm{H}$ II nebula, such as the Orion Blister. The calculations have been done with the development version of CLOUDY (Ferland et al. 2017; nchanging branch, revision r12537).

\subsection{BLR model}

RRL can be used to probe AGNs' continuum source due to their minimal dust attenuation (Scoville \& Murchikova 2013) and to differentiate them from Startburst galaxies by using the ratio of He II to H I intensities from RRL. The RRL from the BLR could be detectable using the capabilities of the SKA radio-telescope (Manti et al. 2016, 2017). In this section, we study how the different data sets affect our simulations of a cloud irradiated by an AGN-type SED. In order to do this, we will first study how the chosen SED and density affect the equilibrium balance, and thus the emission of the RRL. Then, we will analyse the effect of the different data sets on the opacity and intensity of RRLs.

\subsubsection{Dependence of the results on the SED}

Scoville \& Murchikova (2013) simulate the SED of an AGN source by using a power law in agreement with Osterbrock \& Ferland (2006):

$f_{v} \propto v^{-\alpha}$,

where $\alpha=1.7$. For our study, we have used three different SEDs, shown in Fig. 9, in order to check how the incident ionizing flux in the UV affects the Hn $\alpha$ emission and optical depth. The general shape of AGNs SED is described by the SED proposed by Mathews \& Ferland (1987, hereafter M\&F 87), which describes a 'bump' of the incident radiation in the UV, and a cut-off at higher frequencies. A more complicated formula was proposed by Korista et al. (1997, hereafter KBFV 97):

$f_{v}=v^{\alpha_{\mathrm{uv}}} \exp \left(-h v / k T_{\mathrm{BB}}\right) \exp \left(-k T_{\mathrm{IR}} / h v\right)+a v^{\alpha_{\mathrm{X}}}$.

The Big Bump component in the UV is assumed to have an infrared exponential cut-off at $k T_{\mathrm{IR}}=0.01 \mathrm{Ryd}$. The coefficient $a$ is adjusted to produce the correct $\mathrm{X}$-ray to UV ratio. The X-ray power law is only added for energies greater than $1.36 \mathrm{eV}^{3}$ to prevent it from extending into the infrared, where a power law of this slope would produce very strong free-free heating; and less than $100 \mathrm{keV}$. Above $100 \mathrm{keV}$ the continuum is assumed to fall off as $v^{-2}$. For KBFV 97 $T_{\mathrm{BB}}=1.5 \times 10^{5} \mathrm{~K}, \alpha_{\mathrm{uv}}=-0.5$, and $\alpha_{\mathrm{x}}=-1$.

M\&F's and KBFV's SEDs differ from the exponential law model used by Scoville \& Murchikova (2013) in the high-energy region.

\footnotetext{
${ }^{3}$ Here we follow the custom of using eV units for the X-ray spectrum. $1.36 \mathrm{eV}$ correspond to $3.29 \times 10^{5} \mathrm{GHz}$ and $100 \mathrm{KeV}=2.42 \times 10^{10} \mathrm{GHz}$.
} 


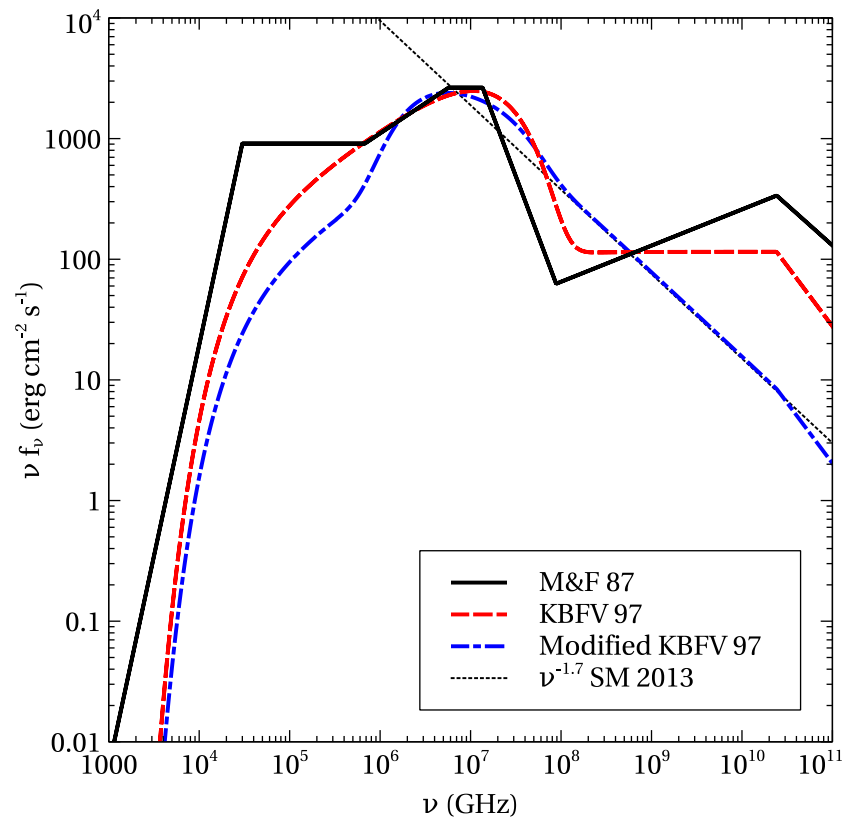

Figure 9. Incident flux $\left(v f_{v}\right)$ used in our BLR models. The legends refer to the following SEDs: M\&F 87: Mathews \& Ferland (1987); KBFV 97: Korista et al. (1997); SM 2013: Scoville \& Murchikova (2013). The assumed ionization parameter is $\log U=-1.5$. For reference, the ionization threshold for hydrogen is $v=3.5 \times 10^{7} \mathrm{GHz}$.

The shape of the high energy cut-off of the different SED could affect the ionization fraction of hydrogen and the length of the ionization phase, so we expect slightly different RRL emission. We have created a different SED to reproduce the $v^{-1.7}$ power law, while conserving the drop of the flux at low frequencies in order to avoid an unrealistic infrared radiation field, using the indices $T_{\mathrm{BB}}=10^{6} \mathrm{~K}$, $\alpha_{\mathrm{uv}}=-0.5$, and $\alpha_{\mathrm{x}}=-1.7$ in equation (22), shown in Fig. 9 as a blue dot-dashed line.

Simulations were carried out for $\mathrm{H}$ atoms with levels up to $n=$ 205 of which $n=1-5$ are $l$-resolved (200 unresolved levels). At the lowest densities considered here, the $n$-shells immediately higher than $n=5$ will not be statistically populated in the $l$-subshells, but we are focused in emission of lines at higher $n \sim 30$ and we do not expect this to affect our results. We have stopped our calculation when the ratio of the electron to hydrogen densities is 0.5 , meaning that half of the hydrogen is recombined. ${ }^{4}$ This ensures that we are obtaining the emission of the ionized phase of the cloud irradiated by the AGN. If a neutral phase exists beyond the ionization front, it might affect the observed intensities and optical depths and depend strongly on the SEDs. This will be the scope of future work.

Relative RRL emission with respect to M\&F 87 SED are shown in Fig. 10 for the different SEDs considered here, at densities $n_{\mathrm{H}}=$ $10^{8} \mathrm{~cm}^{-3}$ and for the different data sets considered here. There is a disagreement of the emission of $\mathrm{H} n \alpha$ lines with respect to the M\&F 87 SED, with a peak localized at $v \sim 10 \mathrm{GHz}$ or $\mathrm{H} 80 \alpha$ for all collisional data sets. This is the same for all data sets. The peak is explained by the amount of the UV photons available that increase the thickness of the column of ionized gas.

In summary, the shape of the assumed SED can translate to differences in the emitted intensities like the ones reported in Fig. 10,

${ }^{4}$ CLOUDY command stop eden 0.5 .

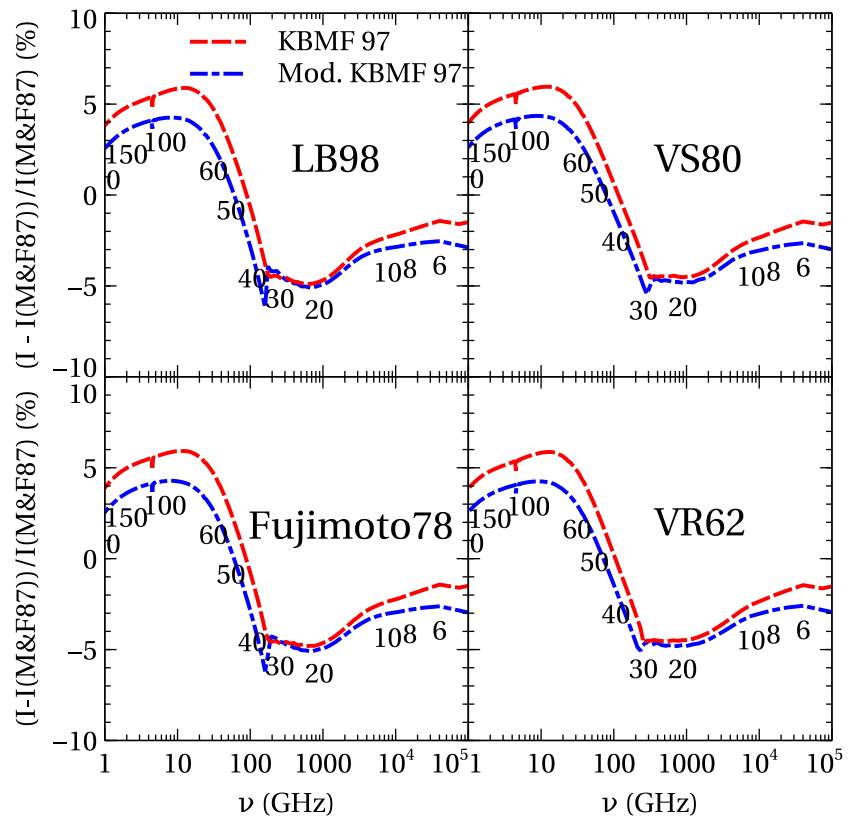

Figure 10. Percentage difference of Hn $\alpha$ RRL emission with respect to the calculations using M\&F 87 SED for the KBFV 97 and the modified KBFV 97 SED shown in Fig. 9. Calculations were done for density $n=10^{8} \mathrm{~cm}^{-3}$. Labels indicate the level $n$ of the Hn $\alpha$ lines. Each panel correspond to each of the different collisional data sets of Fig. 1.

when the UV/X-ray intensity of the SED affects the thickness of the Strömgrem sphere.

\subsubsection{Dependence of the results on the BLR density}

To analyse how the properties of the BLR vary with the gas density $n_{\mathrm{H}}$, we have used the SED from M\&F 87 and LB98 $n$-changing collisional excitation rates, and varied the density of the irradiated gas while keeping the ionization parameter $U$ (Osterbrock \& Ferland 2006) constant. The Hn $\alpha$ RRL emitted intensity (normalized to $\mathrm{H} \beta$ ) is given in Fig. 11 for hydrogen densities running from $n_{\mathrm{H}}=$ $10^{2} \mathrm{~cm}^{-3}$ to $n_{\mathrm{H}}=10^{12} \mathrm{~cm}^{-3}$. For the lowest densities, the curves $n_{\mathrm{H}}=10^{2} \mathrm{~cm}^{-3}, n_{\mathrm{H}}=10^{6} \mathrm{~cm}^{-3}$, and $n_{\mathrm{H}}=10^{8} \mathrm{~cm}^{-3}$ switch their position progressively at $\sim 300 \mathrm{GHz}, \sim 20 \mathrm{GHz}$, and $\sim 2 \mathrm{GHz}$. The origin of this is explained by looking at the optical depths given in Fig. 12. For frequencies longward of $v \sim 200 \mathrm{GHz}$ up to $\sim 2 \times 10^{4}$ $\mathrm{GHz}$, the $\mathrm{H} n \alpha$ transitions are mased for densities $n_{\mathrm{H}}=10^{2}-$ $10^{8} \mathrm{~cm}^{-3}$ which are all in the same order of magnitude (note the 13 orders of magnitude range of Fig. 11) but their intensity ratio with respect to the $\mathrm{H} \beta$ line is bigger according to how strong they are masing. At $\sim 300 \mathrm{GHz}$, the ionized plasma at $n_{\mathrm{H}}=10^{8} \mathrm{~cm}^{-3}$ becomes optically thick and keeps increasing its optical depth up to $\tau=1$ at $\sim 200 \mathrm{GHz}$ coinciding with the inflexion of the intensity curve, which is not amplified anymore by masers, but rather damped by absorption. The same process happens for $n_{\mathrm{H}}=10^{6} \mathrm{~cm}^{-3}$ at $\sim 20 \mathrm{GHz}$ and again at $\sim 1 \mathrm{GHz}$ for $n_{\mathrm{H}}=10^{4} \mathrm{~cm}^{-3}$ (inset of Fig. 11). At $n_{\mathrm{H}} \geq 10^{10} \mathrm{~cm}^{-3}$ there is no masing in this frequency range, so the Hn $\alpha$ lines are not amplified. This is because, at the higher electron densities, collisions bring the levels close to LTE and undo the inversion of the population that causes masing. Note that, for densities $n_{\mathrm{H}}=10^{12} \mathrm{~cm}^{-3}$, the higher absorption is compensated by the higher number of emitting atoms producing a flux higher than the one for $n_{\mathrm{H}}=10^{10} \mathrm{~cm}^{-3}$. 


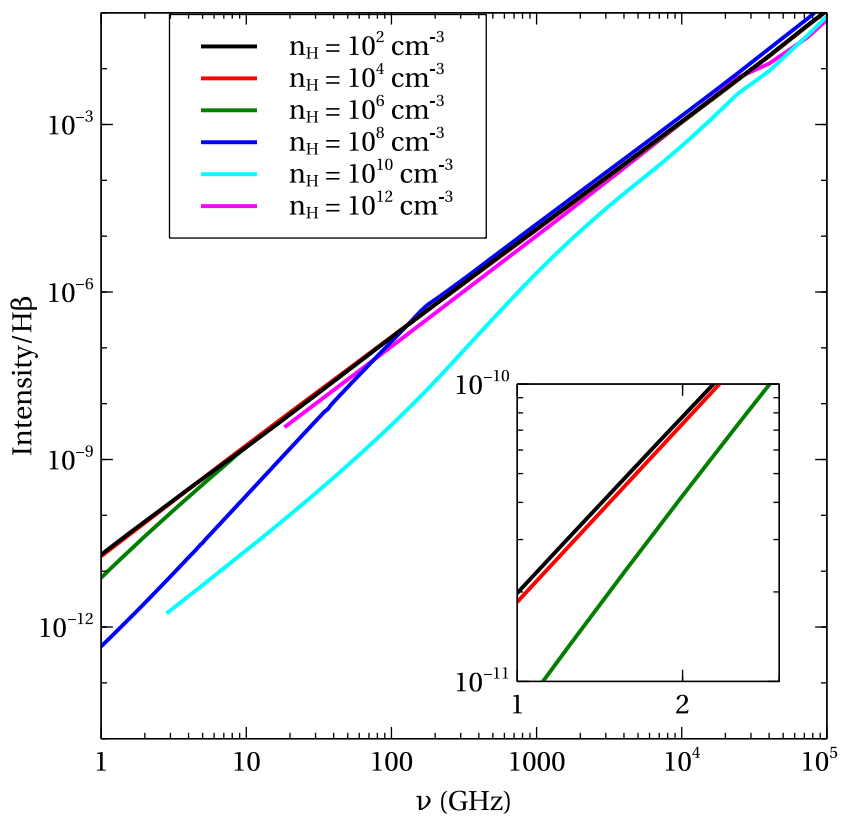

Figure 11. $\mathrm{H} n \alpha$ intensities normalized to $\mathrm{H} \beta$ for a layer of gas irradiated by the M\&F 87 SED of Fig. 9. Collisional excitation data from LB98 data set. At $n_{\mathrm{H}}=10^{10} \mathrm{~cm}^{-3}$ and $n_{\mathrm{H}}=10^{12} \mathrm{~cm}^{-3}$ the curves are truncated due to continuum lowering. In the inset there is an enlargement of the low frequencies region.

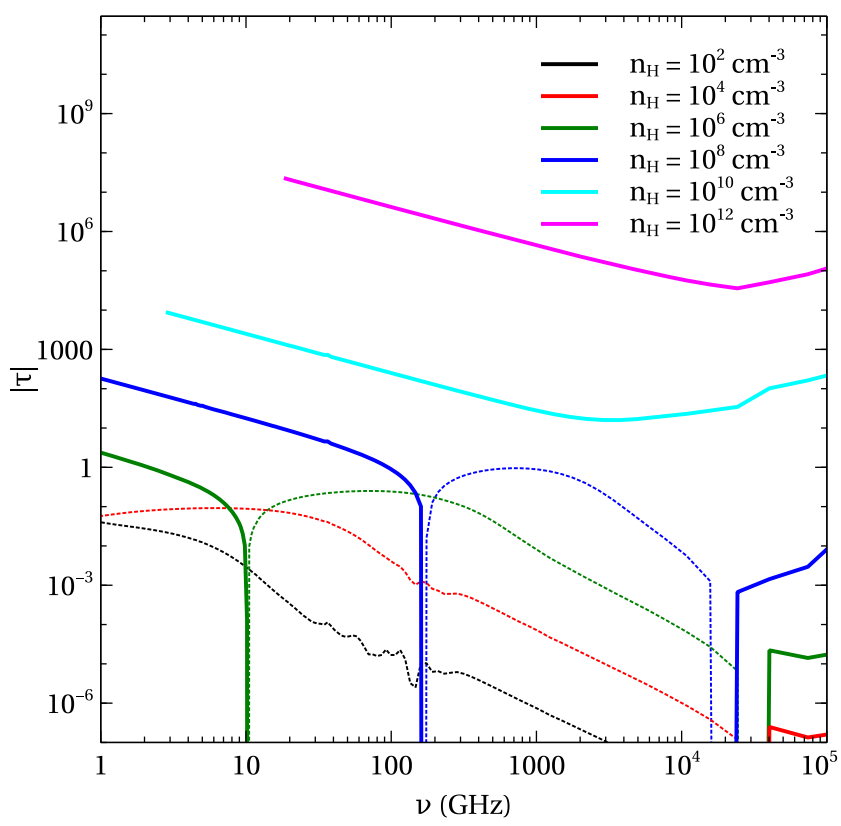

Figure 12. Absolute values of the optical depths at $\mathrm{H} n \alpha$ wavelengths for a layer of gas irradiated by the M\&F 87 SED of Fig. 9. Collisional excitation data from LB98 data set. Dotted lines correspond to the negative values of $\tau$. At $n_{\mathrm{H}}=10^{10} \mathrm{~cm}^{-3}$ and $n_{\mathrm{H}}=10^{12} \mathrm{~cm}^{-3}$ the curves are truncated due to the continuum lowering.

The ranges of negative $\tau$ do not agree with the ones of Scoville \& Murchikova (2013). These authors, use the absorption coefficients provided by Storey \& Hummer (1995), for a range of temperatures and densities. Our calculations using CLOUDY are self-consistent and the temperature and ionization structure are deduced from the radiation field and the density after solving the CR equations (12).

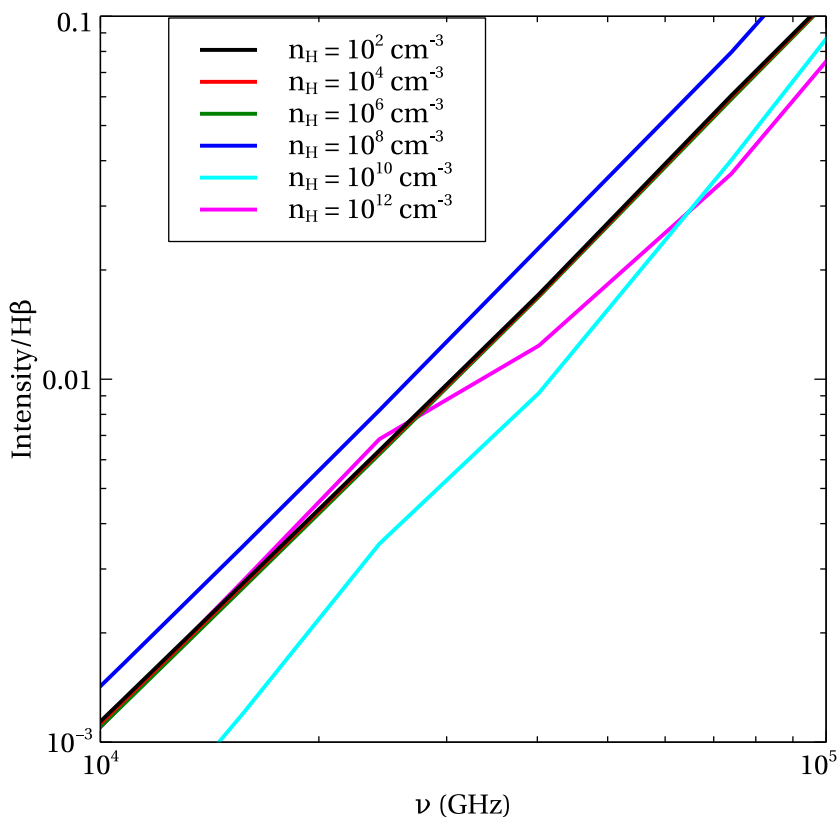

Figure 13. Zoom at high frequencies of Fig. 11.

The cloud is divided in zones and the radiation output from one zone is the input of the next one (see Ferland et al. 2017). The SED distribution in the infrared will contribute to ionize the Rydbergs carrying their population far from LTE.

At high frequencies $v \sim 3 \times 10^{4} \mathrm{GHz}$ the low density curves in Fig. 11 are parallel and there is no change even when the $\mathrm{H} n \alpha$ lines are mased (Fig. 13). The reason for this is that the optical depths are too low and the plasma is optically thin. Therefore, the variation of the optical depth is not enough to produce any changes in the $\mathrm{H} n \alpha$ intensities in this range of frequencies where the number of emitting atoms is determinant.

Continuum Lowering. For $n_{\mathrm{H}}=10^{10} \mathrm{~cm}^{-3}$ and $n_{\mathrm{H}}=10^{12} \mathrm{~cm}^{-3}$ the continuum pressure lowers the atom to the principal quantum numbers $n=132$ and $n=71$, respectively, producing a discontinuity in the emission. Continuum lowering is treated in CLOUDY taking the minimum $n$ given by continuum lowering by particle packing, Debye shielding and Stark broadening of the atomic levels using the formulas suggested by Bautista \& Kallman (2000).

\subsubsection{Dependence of the results on the collisional excitation rate coefficients}

Increasing the value of collisional excitation rates for high $n$ is partially analogous to increasing the density, because the number of collisions is compensated with a greater probability of excitation per collision. However, higher density also increases the opacity to the incident radiation and can produce greater diffuse emission field. This effect cannot be reproduced by changing the values of collisional excitation rates. Nevertheless, the equilibrium produced by redistribution of population in the high Rydberg levels by a more efficient collisional excitation shifts the masing range to lower levels, having an important effect on the emission of RRL. This is indeed what can be seen in Fig. 14, for the different SED considered above. The masing range (thin dashed lines at $n_{\mathrm{H}}=10^{6}-10^{8} \mathrm{~cm}^{-3}$ ) gets down to lower frequencies (higher $n$ of the $\mathrm{H} n \alpha$ lines) for the data sets with lower values of excitation rate coefficients, as their population are far from LTE yet. The electron temperature of 


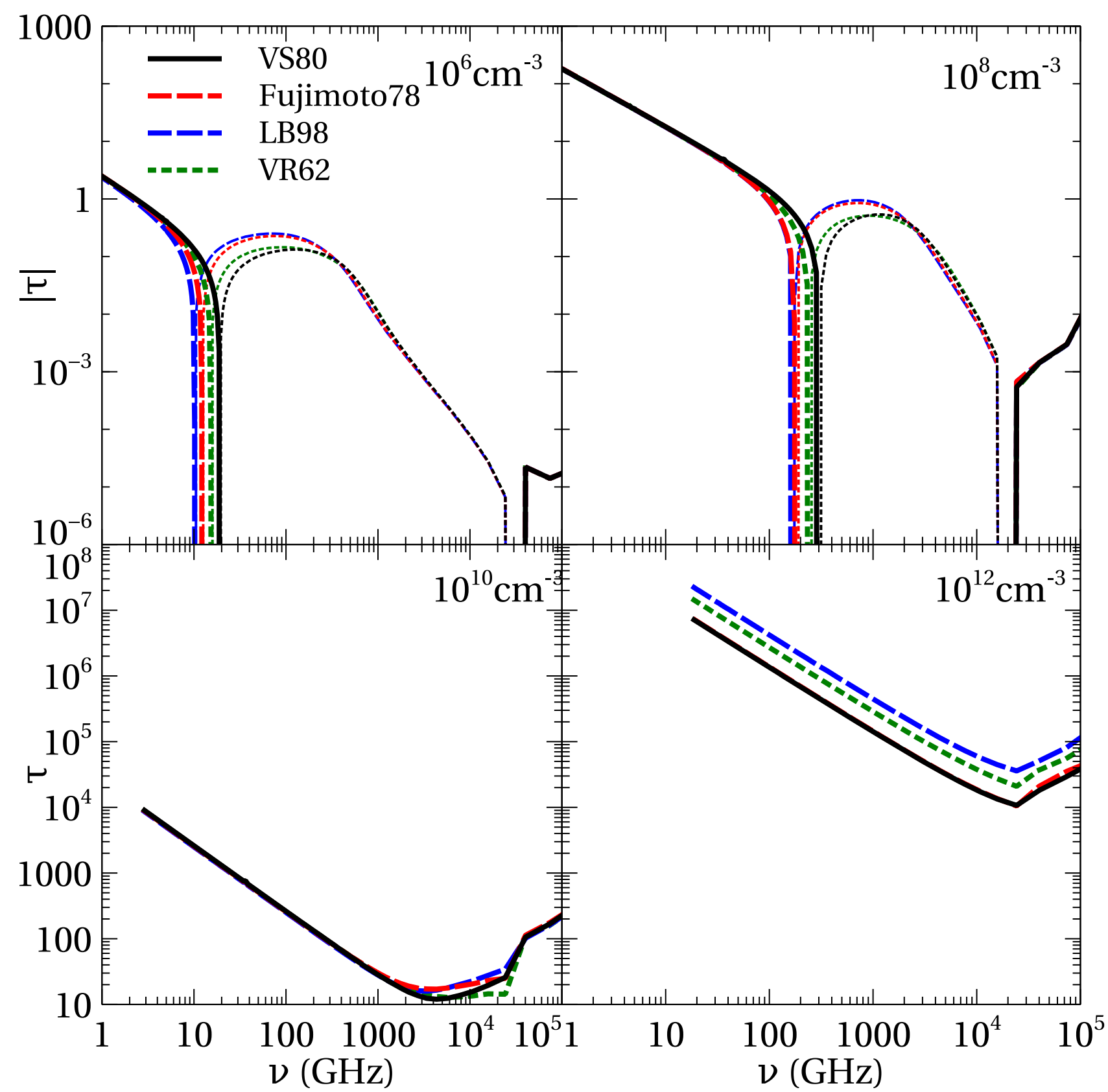

Figure 14. Comparison of the optical depths obtained with the different collisional data sets of Fig. 1 at Hn $\alpha$ wavelengths for a layer of gas irradiated by the M\&F 87 SED of Fig. 9 at different densities. Thick lines correspond to $\tau>0$ while thin lines to $\tau<0$. Labels correspond to the data sets from Fig. 1 .

the fully ionized gas, from where most of the radio recombination emission comes from, is $T_{\mathrm{e}} \sim 15000 \mathrm{~K}$. At higher densities $\left(n_{\mathrm{H}}=\right.$ $10^{10}-10^{12} \mathrm{~cm}^{-3}$ ) the optical depths are so high that most of the levels of the system can be considered to be close to LTE and all the data sets result in parallel curves depending on the differences in collisional rates. Similar results with small variations are obtained for the other SED considered above.

The differences in optical depths propagate to the line emission: in Fig. 15, the percentage differences of the line emission, with respect to the ones obtained using LB98 collisional data, are plotted for different densities. Once again, the deviations on the emissivities of the $\mathrm{H} n \alpha$ lines are linked to the masing range. At densities $n_{\mathrm{H}}=$ $10^{6}-10^{8} \mathrm{~cm}^{-3}$, the differences peak at the frequency where the optical depths change sign from negative to positive. In both cases, for frequencies higher than this point $\left(\sim 20 \mathrm{GHz}\right.$ for $n_{\mathrm{H}}=10^{6} \mathrm{~cm}^{-3}$ and $\sim 200 \mathrm{GHz}$ for $n_{\mathrm{H}}=10^{8} \mathrm{~cm}^{-3}$ ), the higher collisionality of VS80 and VR62 overcompensates the slightly less negative $\tau$, populating the higher levels and producing up to 10 per cent more intense $\mathrm{H} n \alpha$ lines. However, those simulations also have a change of the sign of the optical depths at slightly higher frequencies than the ones using LB98 or Fujimoto78 data. At this point ( $v \sim 300 \mathrm{GHz}$ for VS80), the Hn $\alpha$ lines are still mased for the other data sets (LB98 and Fujimoto78), so they predict higher intensities, up to 20 per cent for $n_{\mathrm{H}}=10^{8} \mathrm{~cm}^{-3}$ and up to 10 percent for $n_{\mathrm{H}}=10^{6} \mathrm{~cm}^{-3}$, respectively, for the range of frequencies down to the frequency point where they have an opacity similar to the other simulations.

For the high densities represented in Figs 14 and 15, the main differences are correlated with the minima of the optical depths, 


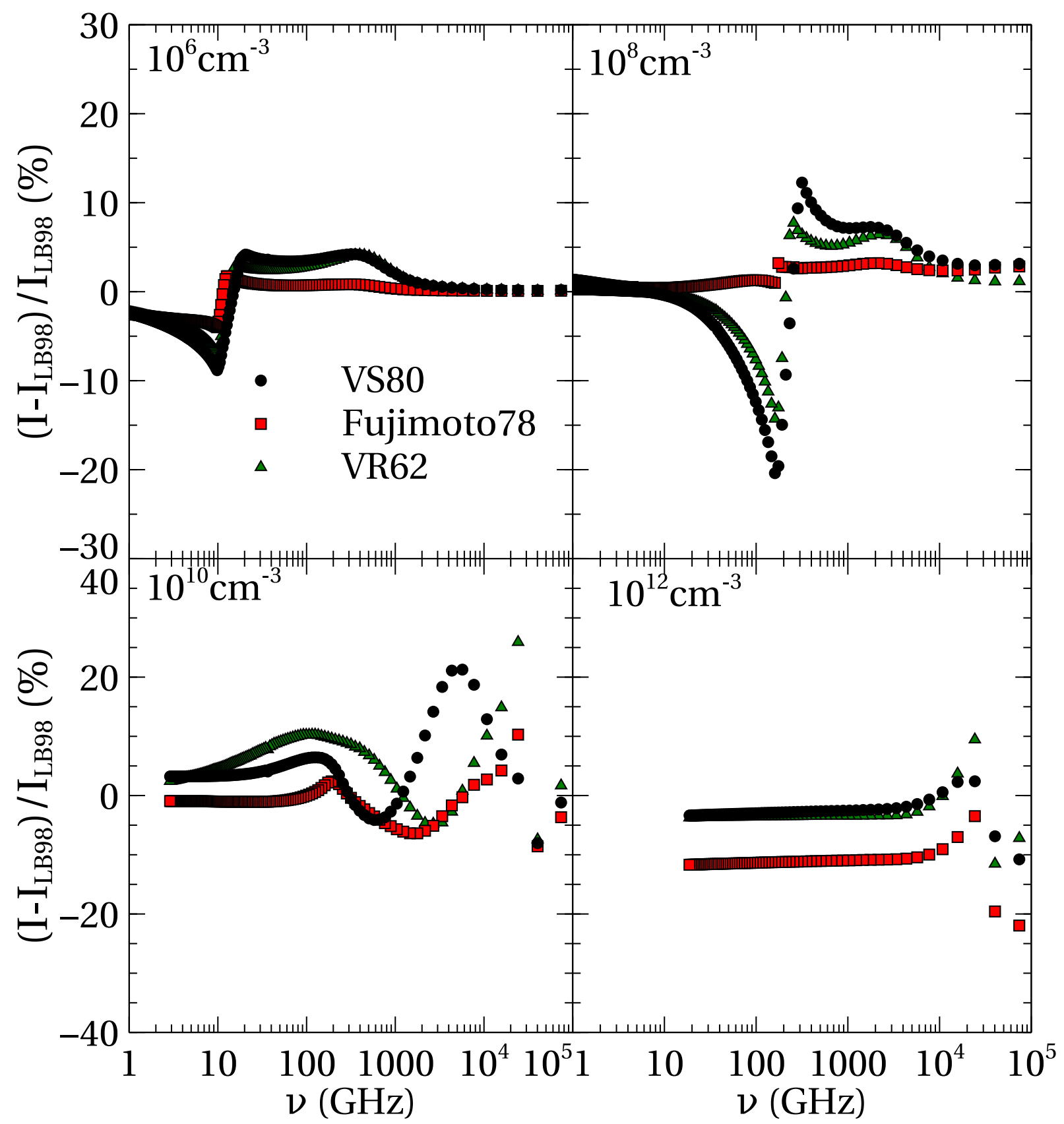

Figure 15. Comparison of the intensities (with respect to LB98) obtained with the different collisional data sets of Fig. 1 at Hno wavelengths for a layer of gas irradiated by the M\&F 87 SED of Fig. 9. Labels correspond to the data sets from Fig. 1.

where the level populations are far away from LTE, and the uncertainties reach 20 percent. At lower frequencies, the curves are parallel and the intensity differences, as the optical depths, are driven by the differences in the values of the effective collisional excitation rates.

\subsection{Orion model}

Our model of the Orion Blister consists of an expanding nebula where the abundance of the species are a mean of the abundances of the Orion Nebula determined by Baldwin et al. (1991), Rubin et al. (1991), Osterbrock, Tran \& Veilleux (1992), and Rubin, Dufour \& Walter (1993). The large R-size grains distribution described by Baldwin et al. (1991) is used, but with the physics updated as described by van Hoof et al. (2004). Abundances of some rare species were taken from the ISM mix of Savage \& Sembach (1996). The ionizing radiation from the $\mathrm{O} 6 \operatorname{star} \theta^{1}$ Orionis $\mathrm{C}$ is simulated from the atmospheres computed by Castelli \& Kurucz (2004). We assumed a homogeneous density of $\sim 10^{4} \mathrm{~cm}^{-3}$ and constant pressure. We have doubled the calculated optical depths in order to account for the molecular cloud beyond the ionization front, which is not simulated. This approximation might look rough, but is not important for the purpose of comparing different collisional rates. Finally the ionizing cosmic rays background is given by Indriolo et al. (2007).

To properly calculate the lines and optical depths of Rydberg levels transitions, we have extended the hydrogen and helium atoms up to $n=400$. The levels up to $n=25$ of H-like hydrogen 
Table 1. Critical densities for $l$-changing processes for $n=20$ and $n=25$ in our Orion nebula model.

\begin{tabular}{lcc}
\hline Ion/Atom & \multicolumn{2}{c}{ Critical density $\left(\mathrm{cm}^{-3}\right)$} \\
& $n=20$ & $n=25$ \\
\hline $\mathrm{H}^{0}$ & 383 & 54 \\
$\mathrm{He}^{0}$ & 194 & 23 \\
$\mathrm{He}^{+}$ & 18903 & 2605 \\
$\mathrm{C}^{4+}$ & $3.136 \times 10^{6}$ & $3.711 \times 10^{5}$ \\
$\mathrm{C}^{5+}$ & $1.911 \times 10^{7}$ & $2.329 \times 10^{6}$ \\
\hline
\end{tabular}

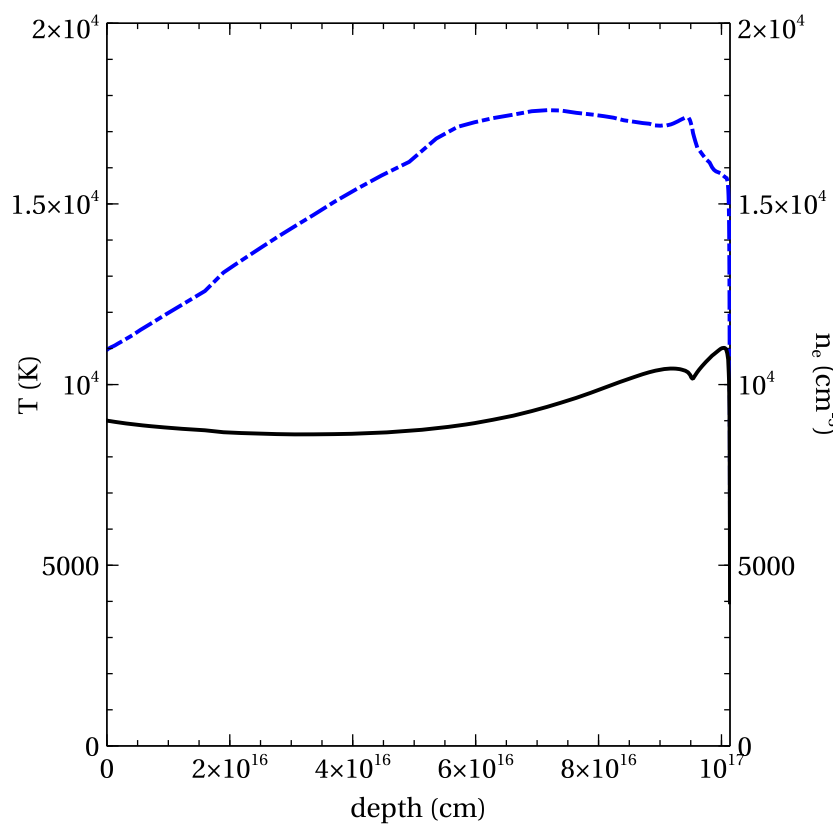

Figure 16. Electron temperature (solid black) and electron density (dotdashed blue) in our Orion Blister model as a function of the depth of the cloud.

and up to $n=20$ of He-like helium are resolved in $l$-subshells, while the $l$ populations of the remaining levels up to $n=400$ are taken as statistically populated. The critical densities for $l$-changing collisions of $n=20$ and $n=25$ are shown in Table 1 for H-like and He-like hydrogen, helium, and carbon. The calculated electron density ranges between $1.09-1.75 \times 10^{4} \mathrm{~cm}^{-3}$ and is shown in Fig. 16. Higher nuclear charge ions, such as carbon ions, have much higher critical densities and are not correctly described, as seen in Table 1. Including more resolved levels for these ions implies more memory and more computational time, which would render the calculation impractical. However, note that the main carbon ionization stages are $\mathrm{C}^{2+}$ (Be-like) in the inner part of the nebula and $\mathrm{C}^{+}$in the outer parts, and $\mathrm{H} / \mathrm{He}$-like $\mathrm{C}$ is negligible in any of the zones. If temperature and radiation conditions would allow for $\mathrm{H} / \mathrm{He}$-like ionized carbon, resolved calculation would be needed for an accurate calculation of the lower levels recombination lines.

In Fig. 17, the intensities emitted at the surface of the cloud for the wavelengths of the $\mathrm{H} n \alpha$ recombination lines are given for calculations using the different data sets presented in Section 2. The percentage difference of the intensities to the ones obtained using the LB98 rate coefficients is given as a function of the frequency.

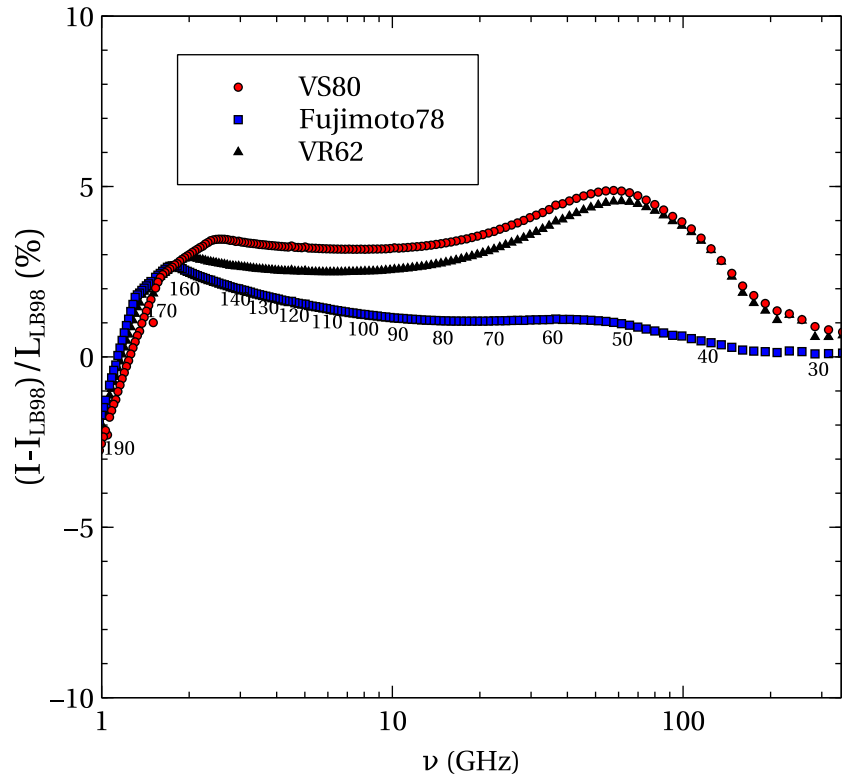

Figure 17. Percentage of the ratios of emitted $\mathrm{H} n \alpha$ transitions with respect to LB98 $n$-changing data set calculations. The small numbers in the figure represent the corresponding $n$ of the Hn$\alpha$ transition. Data sets of the legend correspond to the ones given in Fig. 1.

As seen in the Figure, VS80 and VR62 data sets produce a flux that disagrees with LB98 and Fujimoto78 by 5 per cent between 2 and $300 \mathrm{GHZ}$. This is consistent with the results of Fig. 1. As expected, all data sets tend to produce similar intensities at frequencies around $1 \mathrm{GHz}$ when the system approaches LTE. The shape of the curves of Fig. 17 can be explained with the optical depths, shown in Fig. 18, at the wavelengths of the $\mathrm{H} n \alpha$ lines for the different $n$-changing data sets. The optical depths are negligible for high frequencies down to $\sim 40 \mathrm{GHz}$, where maser emission starts to take place. The temperature in the ionized nebula, shown in Fig. 16, is roughly constant around an average temperature of $8800 \mathrm{~K}$. It corresponds to the zone in Fig. 2 where both Fujimoto 78 and LB98 agree. From Figs 1 and 16, in an optical thin plasma (which is the case here with $\tau \sim 0.1$, it is expected that the difference of intensities stays constant, as the curves in Fig. 1 are parallel for higher $n(n \sim 50)$. However, the progressively less negative $\tau$ of VS80 and VR62 and the sustained value of $\tau$ corresponding to the LB98 data set, due to the maser effect, partially cancels the difference. At frequencies lower than $2 \mathrm{GHz}$, the finite optical depth, that is starting to grow for all data sets, equalizes the fluxes.

The modelled optical depths of Fig. 18 are consistent with what is expected from Fig. 6 and equation (19) (note that the quantities plotted in Fig. 6 are $\log \left(1-\beta_{n}\right)$ ). Even though the model in Fig. 6 is a simplification, the Orion Blister can be approximated as a slab of gas and, if the interaction between $\mathrm{H}$ ions and the other species is neglected (charge exchange cross-sections at these temperatures are much lower than radiative and three body recombination crosssections and the abundances of metals are low ${ }^{5}$ ), our model in Section 3.2 can be seen as a highly simplified optically thin version of the one presented in this section. In Section 3.2, all data sets have a negative value of $\beta_{n}$ up to $n \sim 200$, implying inversion of

${ }^{5} \mathrm{C}$ and $\mathrm{O}$ ions will have an important effect in cooling, however they will not affect the abundances of $\mathrm{H}$. 


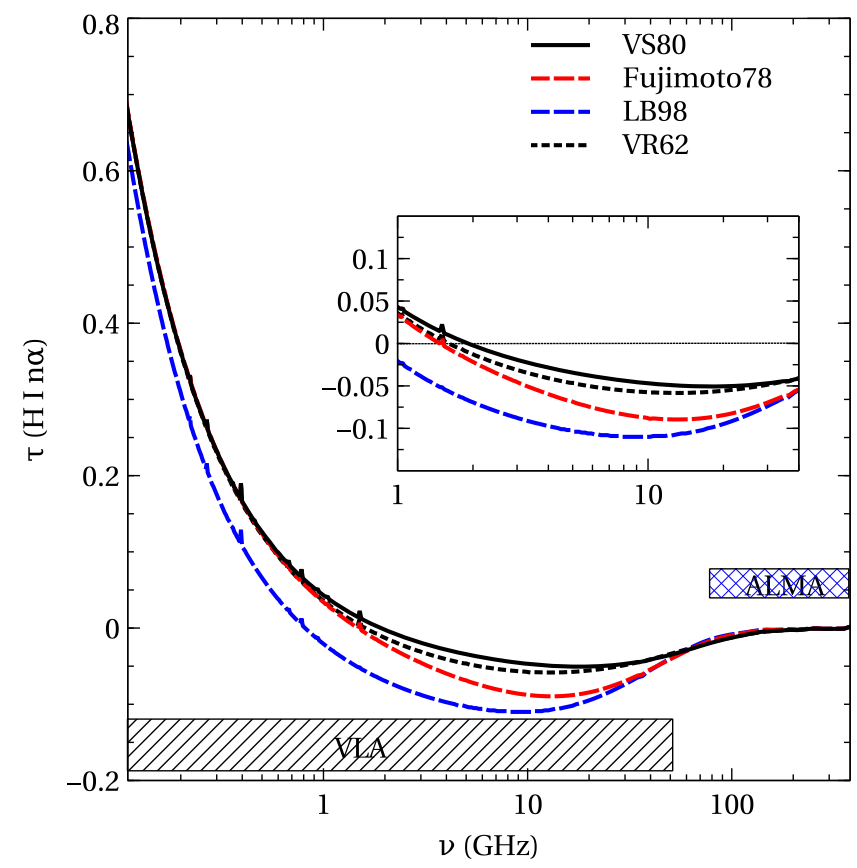

Figure 18. Optical depths at the wavelengths of the Hn $\alpha$ lines. The different symbols correspond to the different data sets in Fig. 1. The inset is a zoom of the $1-40 \mathrm{GHz}$ zone where masing is present. The spikes correspond to the overlapping of different lines. The strongest are: at $1.53 \mathrm{GHz} \mathrm{H} 163 \alpha$, He I $163 \alpha$, and $\mathrm{H} 205 \beta$; at $0.79 \mathrm{GHz} \mathrm{H} 203 \alpha$ and $\mathrm{H} 292 \beta$; at $0.40 \mathrm{GHz} \mathrm{H} 255 \alpha$, $\mathrm{H} 321 \beta$, and $\mathrm{H} 367 \gamma$. The boxes indicate the frequency range of observation of the ALMA and VLA radio telescopes.

the populations and the masing of the $\mathrm{H} n \alpha$ lines at these levels (in Fig. 18 we obtain negative $\tau$ of $\sim-0.05 \rightarrow-0.1$ ). Higher collisional excitation rates, such as those of VS80 and VR62, suppress the masing effect by bringing the population of the Rydberg levels closer to LTE. At low frequencies (high $n$ ), when the populations are close to equilibrium, the optical depths converge to the LTE values. The intensities obtained in our calculations and shown in Figs 17 disagree by less than a 5 percent and they are within the observational uncertainties.

\section{CONCLUSIONS}

In this paper we have presented a comparative study of the effect that the uncertainties in high $n$ collisional data have on the recombination lines of Rydberg atoms and how they affect spectroscopic-derived quantities as line intensities and optical depths.

Of the different data sets analysed here and that are widely used by the astronomical community, two, VS80 (Vriens \& Smeets 1980) and Fujimoto78 (Fujimoto 1978), are based on semi-empirical calculations, one, VR62 (van Regemorter 1962), is the $\bar{g}$ approximation where the Gaunt factors have been fitted to experimental results previous to 1962, and the last one, LB98 (Lebedev \& Beigman 1998), is ab initio impact parameter Born approximation results. All data sets presented here show a reasonable level of agreement, to within a factor $\sim 2$. However, our simulations predict unexpectedly different emission in Hn $\alpha$ RL for some frequency ranges. This is related to the effectiveness of the electron (de-)excitation collisions in the redistribution of the high Rydberg electron population. This changes the level where the $\mathrm{H} n \alpha$ lines stop masing, i.e. the point where the optical depth at the $\mathrm{H} n \alpha$ frequencies changes sign from negative to positive.

In order to check the importance of the collisional excitation uncertainties in the modelling of astronomical objects, we have modelled the RRL emission in BLRs and the Orion Blister H II region. Manti et al. $(2016,2017)$ postulate that RRL from obscured QSO could be easily detectable with the SKA radio-telescope, while Izumi et al. (2016) predict that sub-mm RRL would be too faint to be observed, even with ALMA. However, we have noticed that the latter estimation was done assuming that levels with principal quantum number $n \simeq 20$ would be close to LTE, something that does not happen in our simulations. BLRs physical conditions, and thus its emission, can depend on the energy input (SED) and the density. Differences in the assumed SED changes the length of the ionized region and can produce changes on the emission of $\mathrm{H} n \alpha$. Different densities can determine where the $\mathrm{H} n \alpha$ lines can be mased and produce a systematic offset in the absolute intensity emitted by the cloud. The error introduced by the uncertainty in the collisional data is constrained to a specific frequency range, usually between 40 and $100 \mathrm{GHz}$. Differences on emission $\mathrm{H} n \alpha$ lines can amount up to 20 percent for $n_{\mathrm{H}} \geq 10^{8} \mathrm{~cm}^{-3}$ and 10 per cent for $n_{\mathrm{H}}=10^{6} \mathrm{~cm}^{-3}$. The Orion Blister has been simulated using the model described by Baldwin et al. (1991). We see differences in the optical depths that are reflected in uncertainties of $\sim 5$ per cent in the Hn $\alpha$ RRL which are not relevant for astronomical observations.

Uncertainties of $\sim 60$ percent on the electron impact excitation data propagate to $\sim 5$ percent error on the predicted emission intensities. We do not expect that these differences could be higher than the experimental error. However, in situations where natural masing of the Rydberg levels are expected, these differences can increase up to a significant 20 percent as has been shown here for BLRs. We note that, when neutral gas resides beyond the ionization front of BLRs, $n$-changing collisions could be relevant in the transition zone where the $\mathrm{H}$ and $\mathrm{He}$ are recombining and produce a significant effect on the emitted intensities. Future work will clarify this point.

Accurate collisional data might play a significant role in the determination of the electron fraction and the radiation during the recombination era. Chluba \& Ali-Haïmoud (2016) (in their recombination code COSMOSPEC) use the collisional rates from van Regemorter (1962), while in other calculations (e.g. Grin \& Hirata 2010; Ali-Haïmoud \& Hirata 2011) collisions are neglected. Chluba \& Ali-Haïmoud (2016) report small effects on the recombination spectrum at low frequencies after increasing (de-)excitation collisions two orders of magnitude. However, they point out the necessity of including collisional data for precise computations of the recombination radiation. Collisional data could be important especially at low redshift $(z \lesssim 1000)$ when matter is more diluted and the differences between the collisional rates might be seen in the free electron fraction. $n$-changing collisions bring the higher levels to LTE and suppress the emission of photons from decaying electrons. In addition, population inversion can exist for highly excited levels during the recombination and reionization epochs resulting in local masing in dense forming structures (Grin \& Hirata 2010). These masers could also be strongly affected by energy changing collisions.

\section{ACKNOWLEDGEMENTS}

The authors acknowledge support from the National Science Foundation (AST-1816537) and NASA ATP program (grant number 
17-ATP17-0141). MC acknowledges support from Space Telescope Science Institute (STScI) (HST-AR-14286.001-A and HSTAR-14556.001-A). PvH acknowledges support from the Belgian Science Policy Office through contract no. BR/143/A2/BRASS. NRB acknowledges support from Science and Technology Facilities Council ( STFC) (UK) through the University of Strathclyde APAP Network grant ST/R000743/1. MD and GF acknowledge support from the National Science Foundation (NSF) (AST-1816537), NASA (ATP 17-0141), and STScI (HST-AR-13914, HST-AR15018). The National Radio Astronomy Observatory is a Facility of the Nacional Science Foundation operated under cooperative agreement by Associated University, Inc.

\section{REFERENCES}

Abramowitz M., Stegun I. A., 1965, Handbook of Mathematical Functions with Formulas, Graphs, and Mathematical Tables. Dover Books on Advanced Mathematics, New York: Dover

Ali-Haïmoud Y., Hirata C. M., 2011, Phys. Rev. D, 83, 043513

Anderson H., Ballance C. P., Badnell N. R., Summers H. P., 2000, J. Phys. B: At. Mol. Opt. Phys., 33, 1255

Anderson L. D., Armentrout W. P., Johnstone B. M., Bania T. M., Balser D. S., Wenger T. V., Cunningham V., 2015, ApJS, 221, 26

Anderson L. D., Armentrout W. P., Luisi M., Bania T. M., Balser D. S., Wenger T. V., 2018, ApJS, 234, 33

Baker J. G., Menzel D. H., 1938, ApJ, 88, 52

Baldwin J. A., Ferland G. J., Martin P. G., Corbin M. R., Cota S. A., Peterson B. M., Slettebak A., 1991, ApJ, 374, 580

Ballance C. P., Griffin D. C., Loch S. D., Boivin R. F., Pindzola M. S., 2006, Phys. Rev. A, 74, 012719

Balser D. S., Rood R. T., Bania T. M., Anderson L. D., 2011, ApJ, 738, 27

Balser D. S., Wenger T. V., Anderson L. D., Bania T. M., 2015, ApJ, 806, 199

Bautista M. A., Kallman T. R., 2000, ApJ, 544, 581

Bray I., Burgess A., Fursa D. V., Tully J. A., 2000, A\&AS, 146, 481

Brocklehurst M., 1970a, MNRAS, 148, 417

Brocklehurst M., 1970b, Nature, 225, 618

Brocklehurst M., Salem M., 1977, Comput. Phys. Commun., 13, 39

Brocklehurst M., Seaton M. J., 1972, MNRAS, 157, 179

Brown R. L., Lockman F. J., Knapp G. R., 1978, ARA\&A, 16, 445

Burgess A., Summers H. P., 1976, MNRAS, 174, 345

Castelli F., Kurucz R. L., 2004, in Piskunov N., Weiss W. W., Gray D. F., eds, Proc. IAU Symp. 210, Modelling of Stellar Atmospheres. Kluwer, Dordrecht, p. A20

Chluba J., Ali-Haïmoud Y., 2016, MNRAS, 456, 3494

Dupree A. K., Goldberg L., 1970, ARA\&A, 8, 231

Ferland G. J. et al., 2017, Rev. Mex. Astron. Astrofis., 53, 385

Fujimoto T., 1978, Technical Report IPPJ-AM-8, Semi-empirical Cross Sections and Rate Coefficients for Excitation and Ionization by Electron Collision and Photoionization of Helium. Nagoya University

Gee C. S., Percival L. C., Lodge J. G., Richards D., 1976, MNRAS, 175, 209

Goldberg L., 1966, ApJ, 144, 1225

Griffin D. C., Ballance C. P., 2009, J. Phys. B: At. Mol. Phys., 42, 235201

Grin D., Hirata C. M., 2010, Phys. Rev. D, 81, 083005

Guzmán F., Badnell N. R., Williams R. J. R., van Hoof P. A. M., Chatzikos M., Ferland G. J., 2016, MNRAS, 459, 3498( Paper I)

Guzmán F., Badnell N. R., Williams R. J. R., van Hoof P. A. M., Chatzikos M., Ferland G. J., 2017, MNRAS, 464, 312( Paper II)

Indriolo N., Geballe T. R., Oka T., McCall B. J., 2007, ApJ, 671, 1736

Izotov Y. I., Thuan T. X., Lipovetsky V. A., 1997, ApJS, 108, 1

Izotov Y. I., Thuan T. X., Guseva N. G., 2014, MNRAS, 445, 778

Izumi T., Nakanishi K., Imanishi M., Kohno K., 2016, MNRAS, 459, 3629

Johnson L. C., 1972, ApJ, 174, 227

Johnson L. C., Hinnov E., 1969, Phys. Rev., 187, 143
Korista K., Baldwin J., Ferland G., Verner D., 1997, ApJS, 108, 401( KBFV 97)

Lebedev V. S., Beigman I. L., 1998, Physics of Highly Excited Atoms and Ions. Springer-Verlag, Heidelberg, Germany

Luisi M., Anderson L. D., Balser D. S., Wenger T. V., Bania T. M., 2017, ApJ, 849, 117

Manti S., Gallerani S., Ferrara A., Feruglio C., Graziani L., Bernardi G., 2016, MNRAS, 456, 98

Manti S., Gallerani S., Ferrara A., Greig B., Feruglio C., 2017, MNRAS, 466,1160

Mathews W. G., Ferland G. J., 1987, ApJ, 323, 456( M\&F 87)

Morabito L. K. et al., 2014, ApJ, 795, L33

Olive K. A., Steigman G., Walker T. P., 2000, Phys. Rep., 333, 389

Osterbrock D. E., Ferland G. J., 2006, Astrophysics of Gaseous Nebulae and Active Galactic Nuclei, 2nd. ed. University Science Books, Sausalito, CA

Osterbrock D. E., Tran H. D., Veilleux S., 1992, ApJ, 389, 305

Pengelly R. M., Seaton M. J., 1964, MNRAS, 127, 165

Percival I. C., Richards D., 1976, Adv. At. Mol. Phys., 11, 1

Percival I. C., Richards D., 1978, MNRAS, 183, 329

Peters T., Longmore S. N., Dullemond C. P., 2012, MNRAS, 425, 2352

Poppi S., Tsivilev A. P., Cortiglioni S., Palumbo G. G. C., Sorochenko R. L., 2007, A\&A, 464, 995

Ralchenko Y., Janev R. K., Kato T., Fursa D. V., Bray I., de Heer F. J., 2008, At. Data Nucl. Data Tables, 94, 603

Rohlfs K., Wilson T. L., 2000, Tools of Radio Astronomy. Springer-Verlag, Berlin, Heidelberg, NY

Rubin R. H., Simpson J. P., Haas M. R., Erickson E. F., 1991, ApJ, 374, 564

Rubin R. H., Dufour R. J., Walter D. K., 1993, ApJ, 413, 242

Salgado F., Morabito L. K., Oonk J. B. R., Salas P., Toribio M. C., Röttgering H. J. A., Tielens A. G. G. M., 2017, ApJ, 837, 141

Savage B. D., Sembach K. R., 1996, ARA\&A, 34, 279

Scoville N., Murchikova L., 2013, ApJ, 779, 75

Seaton M. J., 1959, MNRAS, 119, 81

Seaton M. J., 1962, Proc. Phys. Soc., 79, 1105

Shaver P. A., 1980, A\&A, 91, 279

Sinclair R. M., Yoshikawa S., Harries W. L., Young K. M., Weimer K. E., Johnson J. L., 1965, Phys. Fluids, 8, 118

Storey P. J., Hummer D. G., 1995, MNRAS, 272, 41

van Hoof P. A. M., Weingartner J. C., Martin P. G., Volk K., Ferland G. J., 2004, MNRAS, 350, 1330

van Regemorter H., 1962, ApJ, 136, 906

Vriens L., Smeets A. H. M., 1980, Phys. Rev. A, 22, 940

\section{APPENDIX: IMPORTANCE OF A COMPLETE MODEL}

Maser effects occur for non-LTE RRL around sub-mm frequencies. This affects ALMA measurements (Peters, Longmore \& Dullemond 2012). The simulations of RRL spectra need to include a high number of Rydberg levels, and many of them will not be in LTE for standard cloud densities (see Fig. 4). The CLOUDY simulations used throughout this paper were done by setting the model atom up to high $n$. In an ideal atom, the highest levels (at $n \rightarrow \infty$ ) are in LTE with the continuum and neighbouring levels. However, an infinite levels atom is impractical in models. In CLOUDY, we can use a 'top-off' where the highest level considered is forced in LTE with the continuum by enhancing its collisional ionization and three body recombination. This is an imperfect treatment of the atom and can lead to errors in the populations. These errors can be reduced by the fact that at intermediate densities the continuum is lowered to finite $n$ by the effect of electron pressure (Bautista \& Kallman 2000). To illustrate the lack of accuracy in the high $n$ populations, we have used a simple model of a slab of pure hydrogen gas at $T=7500 \mathrm{~K}$ and $n_{\mathrm{H}}=10^{5} \mathrm{~cm}^{-3}$, divided in 100 zones 

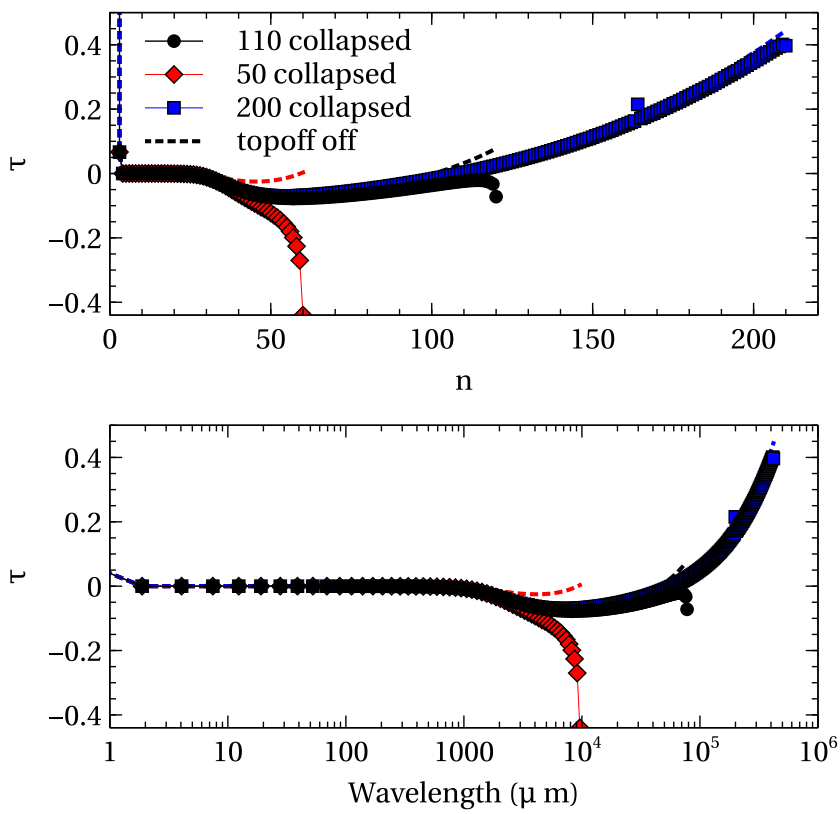

Figure A1. Variation of the optical depth of the Hna lines for a slab of pure hydrogen gas irradiated by blackbody radiation at $T=10^{5} \mathrm{~K}$ including a different number of levels. Top: Optical depth versus highest principal quantum number. Bottom: Optical depth versus wavelength. and irradiated by a blackbody radiation at $T=10^{5} \mathrm{~K}$. We have then calculated the optical depth for different atom sizes with a different number of collapsed levels. The results for the optical depth are in Fig. A1. When the number of levels is not big enough, the enhanced population of the top level produces an increment of the population inversion and an artificial increase of the line emission. If the 'top-off' is disabled, the effect is the contrary as the population of the top level does not account for the cascade from higher levels. The real solution dwells between these extremes. When more and more levels are included in the calculation, the ionization and three body recombination coefficients bring the top level closer to LTE and the effect of the 'top-off' mechanism is diluted.

This paper has been typeset from a $\mathrm{T}_{\mathrm{E}} \mathrm{X} / \mathrm{L} \mathrm{T}_{\mathrm{E}} \mathrm{X}$ file prepared by the author. 